MATHEMATICS OF COMPUTATION

Volume 74, Number 250, Pages 681-708

S 0025-5718(04)01703-X

Article electronically published on August 23, 2004

\title{
DATA-SPARSE APPROXIMATION TO A CLASS OF OPERATOR-VALUED FUNCTIONS
}

\author{
IVAN P. GAVRILYUK, WOLFGANG HACKBUSCH, AND BORIS N. KHOROMSKIJ
}

\begin{abstract}
In earlier papers we developed a method for the data-sparse approximation of the solution operators for elliptic, parabolic, and hyperbolic PDEs based on the Dunford-Cauchy representation to the operator-valued functions of interest combined with the hierarchical matrix approximation of the operator resolvents. In the present paper, we discuss how these techniques can be applied to approximate a hierarchy of the operator-valued functions generated by an elliptic operator $\mathcal{L}$.
\end{abstract}

\section{INTRODUCTION}

In the papers [12]-[15] and [10], a class of hierarchical matrices $(\mathcal{H}$-matrices) has been analysed which are data-sparse and allow an approximate matrix arithmetic with almost linear complexity.

In the present paper, we apply the $\mathcal{H}$-matrix technique to approximate different classes of mappings generated by integrals of functions of an elliptic operator $\mathcal{L}$. These mappings are of the form function-to-operator, operator-to-operator or sequence of operator-to-operator.

As examples of function-to-operator mappings we consider the solution operators to parabolic PDEs (the operator exponential), to elliptic PDEs (the normalised hyperbolic sine function) and to hyperbolic PDEs (the operator cosine function), where these operators are represented by the Dunford-Cauchy integral (cf. [5]-8]). Approximating this integral by a proper quadrature formula (Sinc quadrature or Gauss-Lobatto quadrature) and applying the $\mathcal{H}$-matrix arithmetics to the discrete resolvents lead to a data-sparse representation to the solution operator of interest.

As an example of an operator-to-operator mapping we consider the solution operator to the Lyapunov equation. We use two integral representations of the solution operator, namely (i) by a double Dunford-Cauchy integral and (ii) by an improper integral with the operator exponential. In both cases, the appropriate exponentially convergent quadrature formulae and the $\mathcal{H}$-matrix approximations to the elliptic resolvents or to the operator exponential lead to data-sparse approximations with the linear-logarithmic cost.

As an example of a sequence of operators-to-operator mapping we discuss the solution operator to the Riccati equation by an iterative scheme involving the solution

Received by the editor April 10, 2003.

2000 Mathematics Subject Classification. Primary 65F50, 65F30; Secondary 15A24, 15A99.

Key words and phrases. Operator-valued function, data-sparse approximation, elliptic operator, $\mathcal{H}$-matrices. 
of Lyapunov-Sylvester equations in each step. Together with data-sparse approximations to these solutions, we arrive at algorithms of almost linear complexity.

Note that the data-sparse $\mathcal{H}$-matrix approximation of almost optimal complexity to the operator-valued functions

$$
\begin{aligned}
\mathcal{F}_{1}(\mathcal{L}) & :=e^{-t \mathcal{L}} \\
\mathcal{F}_{2}(\mathcal{L}) & :=\mathcal{L}^{-\alpha}, \alpha>1, \\
\mathcal{F}_{3, k}(\mathcal{L}) & :=\cos (t \sqrt{\mathcal{L}}) \mathcal{L}^{-k}, \quad k \in \mathbb{N}, \\
\mathcal{F}_{4}(\mathcal{L}) & :=\int_{0}^{\infty} e^{t \mathcal{L}^{*}} G e^{t \mathcal{L}} d t,
\end{aligned}
$$

of an elliptic operator $\mathcal{L}$ was addressed in [7] (see $\$ 3$ for more details). In this paper we derive a new quadrature rule for the operator $\mathcal{F}_{4}(\mathcal{L})$ which is more efficient than the previous one from [5, 7]. The method is now based on a double integral representation to the solution operator for equations with the general family of so-called elementary operators (cf. [27]).

In $\$ \$ 3.13 .2$ we are looking for a data-sparse approximation of the solution $X$ to the operator equation

$$
\sum_{j=1}^{M} U_{j} X V_{j}=Y
$$

for certain operators $U_{j}, V_{j}$ and $Y$.

Finally (see 44), we construct an explicit approximation by resolvents to the operator sign-function which can be applied, for example, to represent the solution operator of the algebraic Riccati equation. A short version of this paper was published in [8].

\section{GoAls AND OVERVIEW}

2.1. Hierarchy of the operator-valued functions. In this section we define a hierarchy of operator-valued functions which can be represented by various mappings generated by an elliptic operator $\mathcal{L}$ in a Banach space $X$. In the following, we will develop various discretisations to these mappings. The hierarchy of operatorvalued functions consists of function-to-operator mappings, operator-to-operator and sequence of operators-to-operator mappings which arise in applications related to partial differential equations, control theory and linear algebra.

One basic function of an elliptic operator $\mathcal{L}$ is the inverse $\mathcal{L}^{-1}$. A fast implementation of $\mathcal{L} \mapsto \mathcal{L}^{-1}$ is of interest in finite-element methods for elliptic and parabolic problems. On the other hand, the data-sparse approximation of $\mathcal{L}^{-1}$ plays a central role in our further constructions. However, this topic is already addressed in [12, 13, 14, 10], where the modern $\mathcal{H}$-matrix approximation technique has been presented.

2.1.1. Functions of the first level. Let $\Gamma_{S} \subset \mathbb{C}$ denote a path enveloping the spectrum of $\mathcal{L}$ and let $\Gamma_{I}$ be a path which envelopes but does not intersect $\Gamma_{S}$, for a given function which is analytic inside of $\Gamma_{I}$ (the subscript $I$ abbreviates "integration"). Below, we write $\Gamma$ instead of $\Gamma_{I}$. We can define a bounded operator

$$
F(\mathcal{L})=\frac{1}{2 \pi i} \int_{\Gamma} F(z)(z I-\mathcal{L})^{-1} d z
$$


provided that this Dunford-Cauchy integral converges. The above integral defines a function-to-operator mapping $F(\cdot) \rightarrow F(\mathcal{L})$ generated by a fixed elliptic operator $\mathcal{L}$.

As a first example of such a mapping we consider the solution operator

$$
T(t)=e^{-\mathcal{L} t}=\int_{\Gamma} e^{-z t}(z I-\mathcal{L})^{-1} d z
$$

to the initial-value problem

$$
u^{\prime}(t)+\mathcal{L} u(t)=0, \quad u(0)=u_{0},
$$

where $\mathcal{L}$ is a strongly $P$-positive operator in a Banach space $X$ and $u(t)$ is a vectorvalued function $u: \mathbb{R}_{+} \rightarrow X$ (see [5] for more details). Given the solution operator and the initial vector $u_{0}$, the solution of the initial-value problem can be represented by $u(t)=T(t) u_{0}$. As a simple example of a partial differential equation which can be described by (2.1), one can consider the classical heat equation

$$
\frac{\partial u}{\partial t}-\frac{\partial^{2} u}{\partial x^{2}}=0
$$

with corresponding boundary and initial conditions, where the operator $\mathcal{L}$ is defined by

$$
\begin{aligned}
D(\mathcal{L}) & =\left\{v \in H^{2}(0,1): v(0)=0, v(1)=0\right\}, \\
\mathcal{L} v & =-\frac{d^{2} v}{d x^{2}} \quad \text { for all } v \in D(\mathcal{L}) .
\end{aligned}
$$

Our second example deals with the boundary-value problem

$$
\frac{d^{2} u}{d x^{2}}-\mathcal{L} u=0, \quad u(0)=0, \quad u(1)=u_{1},
$$

in a Banach space $X$ (see [6]). The solution operator is the normalised hyperbolic operator sine family

$$
E(x) \equiv E(x ; \mathcal{L})=(\sinh (\sqrt{\mathcal{L}}))^{-1} \sinh (x \sqrt{\mathcal{L}}),
$$

so that $u(x)=E(x) u_{1}$. This function $E(x)$ is the result of the function-to-operator mapping

$$
(\sinh (\sqrt{\cdot}))^{-1} \sinh (x \sqrt{\cdot}) \rightarrow E(x ; \mathcal{L})
$$

generated by the operator $\mathcal{L}$. The simplest PDE from the class (2.2) is the Laplace equation in a cylindric domain:

$$
\begin{aligned}
\frac{d^{2} u}{d x^{2}}+\frac{d^{2} u}{d y^{2}} & =0, & & x \in[0,1], y \in[c, d], \\
u(0, y) & =0, & & u(1, y)=u_{1}(y) .
\end{aligned}
$$

In the third example we consider the following initial-value problem for the second order differential equation with an operator coefficient:

$$
u^{\prime \prime}(t)+\mathcal{L} u(t)=0, \quad u(0)=u_{0}, \quad u^{\prime}(0)=0,
$$

with the solution operator (the operator cosine family)

$$
C(t ; \mathcal{L})=\cos (t \sqrt{\mathcal{L}})=\int_{\Gamma} \cos (t \sqrt{z})(z I-\mathcal{L})^{-1} d z,
$$


which represents the function-to-operator mapping $\cos (t \sqrt{\cdot}) \rightarrow C(t ; \mathcal{L})$ (see [7] for more details). The simplest example of PDEs from this class is the classical wave equation

$$
\frac{\partial^{2} u}{\partial t^{2}}-\frac{\partial^{2} u}{\partial x^{2}}=0
$$

subject to the corresponding boundary and initial conditions.

2.1.2. Functions of the second level. The next hierarchy level is formed by the operators-to-operator mappings. Let $\left\{\mathcal{G}_{0}(t): t \in\left[t_{0}, t_{1}\right]\right\}$ be an operator family in $X$ and let the integral

$$
\mathcal{F}=\int_{t_{0}}^{t_{1}} \mathcal{G}_{0}(t) d t
$$

exist. Then this integral represents an operators-to-operator mapping $\mathcal{G}_{0} \mapsto \mathcal{F}$. As an example we consider the Sylvester equation

$$
A X+X B=G, \quad(A, B, G \text { given })
$$

with the solution $X$ given by the integral over $\mathcal{G}_{0}(t):=e^{-t A} G e^{-t B}$,

$$
\mathcal{F}(G ; A, B)=\int_{0}^{\infty} e^{-t A} G e^{-t B} d t,
$$

where we suppose that $A, B$ are such that this integral exists. A particular case is the Lyapunov equation

$$
\mathcal{L} X+X \mathcal{L}^{*}=G
$$

with the solution

$$
\mathcal{F}(G ; \mathcal{L})=\int_{0}^{\infty} e^{-t \mathcal{L}^{*}} G e^{-t \mathcal{L}} d t
$$

generated by an (elliptic) operator $\mathcal{L}$.

2.1.3. Functions of the third level. On the next hierarchy level, one can consider a sequence of operators-to-operator mappings which arises for example in the case of the (nonlinear) Riccati equation

$$
A X+X A^{\top}+X F X=G,
$$

where $A, F, G \in \mathbb{R}^{n \times n}$ are given and $X \in \mathbb{R}^{n \times n}$ is the unknown matrix. This equation is of fundamental importance in many applications in control theory. There are numerous methods to solve (2.3) (see, e.g., [1] and the literature therein) and one of the best is based on the matrix function $\operatorname{sign}(H)$. An alternative method is based on Newton's iteration. At each iteration step the Lyapunov equation

$$
\left(A-F X_{n}\right) X_{n+1}+X_{n+1}\left(A-F X_{n}\right)^{\top}=-X_{n} F X_{n}+G:=G_{n}
$$

has to be solved. Assuming the convergence $X_{n} \rightarrow X$, we have the sequence of operators-to-operator mapping

$$
X_{n} \rightarrow X_{n+1} \rightarrow \cdots \rightarrow X:=\mathcal{F}(F, G, A)
$$

of the kind

$$
X=\lim _{n \rightarrow \infty} X_{n}, \quad X_{n+1}:=\int_{0}^{\infty} e^{-t\left(A-F X_{n}\right)} G_{n} e^{-t\left(A-F X_{n}\right)^{\top}} d t .
$$

Under usual assumptions on the data, the Newton method converges quadratically. 
We discuss in more details an algorithm based on the application of the matrix sign-function, which can be defined by

$$
\operatorname{sign}(H)=\frac{1}{\pi i} \int_{\Gamma_{I}}(z I-H)^{-1} d z-I
$$

with $\Gamma_{I}$ being any simply closed curve in the complex plane whose interior contains all eigenvalues of $H$ with positive real part. We require that $H$ have no eigenvalues on the imaginary axis. Rather general integral representations to the matrix $\operatorname{sign}(H)$ were introduced in [16].

An equivalent definition uses the canonical Jordan decomposition $H=Y J Y^{-1}$ of $H$. Let the diagonal part of $J$ be given by the matrix $D=\operatorname{diag}\left(d_{1}, \ldots, d_{n}\right)$. Set $S=\operatorname{diag}\left(s_{1}, \ldots, s_{n}\right)$ with

$$
s_{i}=\left\{\begin{array}{ll}
+1 & \text { if } \Re e\left(d_{i}\right)>0 \\
-1 & \text { if } \Re e\left(d_{i}\right)<0
\end{array}\right\} .
$$

Then we define $\operatorname{sign}(H)=Y S Y^{-1}$.

The following algorithm gives the solution to the Riccati equation by means of the sign function:

1. For $H=\left(\begin{array}{cc}A & M \\ R & -A^{\top}\end{array}\right)$ determine $\left(\begin{array}{cc}W_{11} & W_{12} \\ W_{21} & W_{22}\end{array}\right):=\operatorname{sign}(H)$.

2. Find $X$ as the solution of the minimisation problem (say, by the least squares method)

$$
\min \left\|\left(\begin{array}{c}
W_{12} \\
W_{22}+I
\end{array}\right) X-\left(\begin{array}{c}
I+W_{11} \\
W_{21}
\end{array}\right)\right\|
$$

In $\$ 4$ we propose some exponentially convergent quadrature formulae for the Dunford-Cauchy integral in (2.4) which is built by a sum of resolvents $\left(z_{k} I-H\right)^{-1}$. In this way, the data-sparse solution to the Riccati equation can be based on the $\mathcal{H}$-matrix approximation of the inversion operator.

2.2. Toward approximations of optimal complexity. For the numerical treatment, the operator $\mathcal{F}$ of interest has to be approximated by $n \times n$ matrices. In our approach, we are looking for a family of data-sparse matrices (more specifically, $\mathcal{H}$-matrices) $M_{i} \in \mathbb{R}^{n \times n}$, such that (with a proper projection $\mathcal{P}_{n}: X \rightarrow \mathbb{R}^{n}$ ) the error satisfies the estimate

$$
\phi(N):=\left\|\mathcal{P}_{n} \mathcal{F} \mathcal{P}_{n}^{*}-\sum_{i=1}^{N} M_{i}^{-1}\right\| \leq \varepsilon
$$

in the corresponding operator norm. The parameter $n \in \mathbb{N}$ can be viewed as $n=\operatorname{dim}\left(V_{h}\right)$, where $V_{h}$ is used for the Galerkin approximation of the related elliptic $\mathrm{PDE}$ with a given tolerance.

We require that the class of matrices approximating the operator-valued function allows an almost linear cost estimate by $\mathcal{O}\left(n \log ^{q} n\right)$ for the approximate matrix arithmetic and the memory. Clearly, the inversion of a general $n \times n$-matrix has a complexity of at least $\mathcal{O}\left(n^{2}\right)$. Here, however, we consider the class of matrices arising from FEM and BEM applications. Then the new concept of data-sparse approximations can be applied based on so-called hierarchical matrices $(\mathcal{H}$-matrices) [12, $13,14,10$. The almost linear complexity of the $\mathcal{H}$-matrix arithmetic yields a cost of $\sum M_{i}^{-1}$ bounded by $\mathcal{O}\left(N n \log ^{q} n\right)$ arithmetic operations. 
Due to $n$-width arguments for analytic functions (see, e.g., [1] and references therein), we need $\mathcal{O}(\log 1 / \varepsilon)$ parameters for their $\varepsilon$-approximation (say, by polynomials or Sinc functions). In order to get a polynomial operation count with respect to $\log 1 / \varepsilon$, we would like to ensure that $N=\mathcal{O}\left(\log ^{q} 1 / \varepsilon\right)$, i.e., $\phi(N)$ must be exponential in $N$ (e.g., $\left.\phi(N) \leq c \exp \left(-\gamma N^{\alpha}\right), \alpha, \gamma>0\right)$. In our applications we approximate the analytic function $\mathcal{F}=\mathcal{F}(\mathcal{L})$ for an elliptic operator $\mathcal{L}$ by a sum $\sum_{i=1}^{N} c_{i}\left(z_{i} I-\mathcal{L}\right)^{-1}$ of $N$ elliptic resolvents, such that the sum converges exponentially, i.e., $N=\mathcal{O}\left(\log ^{q} 1 / \varepsilon\right)$. Furthermore, in conventional FEM, the operator $\mathcal{L}$ itself can be approximated by a sparse $n \times n$ stiffness matrix $\mathcal{L}_{h}$ with $\mathcal{O}(n)$ nonzero entries, which leads to the $\mathcal{H}$-matrix inverse $\left(z_{i} I-\mathcal{L}_{h}\right)^{-1}$ with arithmetical costs of $\mathcal{O}\left(n \log ^{p} n\right)$. Therefore, our final complexity bound for the approximation ansatz $\sum M_{i}^{-1}$ leads to $\mathcal{O}\left(n \log ^{q}(1 / \varepsilon) \log ^{p} n\right)$ arithmetical operations.

In the present paper, our goals are

- representation of the mentioned operator-valued functions by an exponentially converging sum of elliptic resolvents,

- construction of well-parallelisable algorithms with almost linear complexity,

- discussion of some applications (PDEs, control problems).

2.3. Integral representation to operators of $\left(f_{S}, f_{R}\right)$-type. Let $A: X \rightarrow X$ be a linear densely defined closed operator in $\mathrm{X}$ with the spectral set $s p(A)$. In this paper, we restrict ourselves to a class of operators of $\left(f_{S}, f_{R}\right)$-type to be defined below. Let $\Gamma_{S}$ be a curve in the complex plane $z=\xi+i \eta$ defined by the equations $\xi=\phi_{S}(s), \eta=\psi_{S}(s), s \in(-\infty, \infty)$ in the coordinates $\xi, \eta$. We denote by

$$
\Omega_{\Gamma_{S}}:=\left\{z=\xi+i \eta: \xi>f_{S}(\eta)\right\}
$$

the domain inside of $\Gamma_{S}$. In what follows, we suppose that this curve lies in the right half-plane of the complex plane and that it contains $s p(A)$, i.e., $s p(A) \subset \Omega_{\Gamma_{S}}$.

The form of the curve enveloping the spectrum of $A$ and the behaviour of the resolvent as a function of $z$ contain important information about the operator $A$ and allow us to develop a calculus of functions of $A$ (cf. [4, 5, 6, 7], 9]).

Definition 2.1. Given an operator $A: X \rightarrow X$, let $f_{S}(\cdot)$ and $f_{R}(\cdot)$ be functions such that

$$
\left\|(z I-A)^{-1}\right\|_{X \rightarrow X} \leq f_{R}(z) \quad \text { for all } z \in \mathbb{C} \backslash \Omega_{\Gamma_{S}} .
$$

Note that $\Gamma_{S}$ is defined by means of $f_{S}$ (cf. (2.5)). Then we say that the operator $A: X \rightarrow X$ is of $\left(f_{S}, f_{R}\right)$-type.

Note that a strongly $P$-positive operator (defined in [4]) is also an operator of $\left(f_{S}, f_{R}\right)$-type with the special choice

$$
f_{S}(\eta)=a \eta^{2}+\gamma_{0}, \quad f_{R}(z)=M /(1+\sqrt{|z|}), \quad a>0, \quad \gamma_{0}>0, \quad M>0 .
$$

In particular, strongly elliptic partial differential operators are strongly $P$-positive.

Let $\mathcal{L}$ be a linear, densely defined, closed operator of $\left(f_{S}, f_{R}\right)$-type in a Banach space $X$. We choose an integration curve $\Gamma_{I}:=\left\{z=\xi+i \eta: \xi=\phi_{I}(s), \eta=\psi_{I}(s)\right\}$ enveloping the so-called "spectral curve" $\xi=f_{S}(\eta)$ (see Figure 1). Let $F(z)$ be a complex-valued function that is analytic inside of the integration curve $\Gamma_{I}$. The next simple theorem offers conditions under which one can define a bounded operator $F(\mathcal{L})$. 


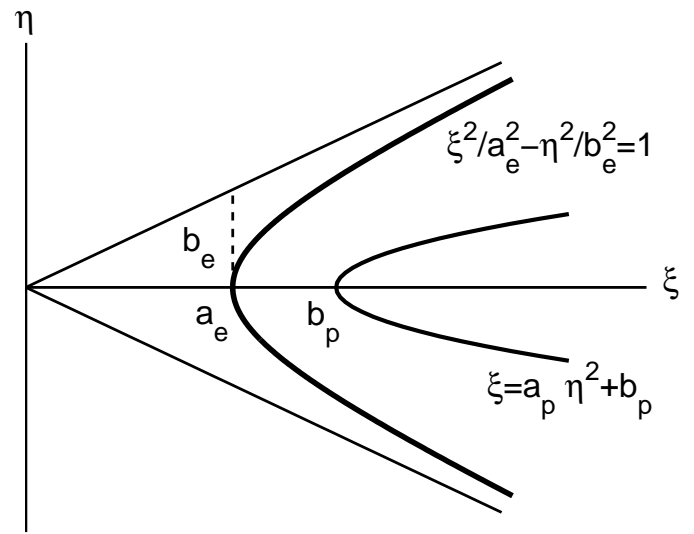

Figure 1. The spectral curve $\Gamma_{S}$ and the integration curve $\Gamma_{I}$.

Theorem 2.2. Let $\xi=f_{I}(\eta)$ be a function defined parametrically by $\xi=\phi_{I}(s), \eta=$ $\psi_{I}(s)$ and assume that the improper integral

$\int_{-\infty}^{\infty}\left|\Phi_{1}(s) f_{R}\left(\phi_{I}(s)+i \psi_{I}(s)\right)\right| d s \quad$ with $\quad \Phi_{1}(s)=F\left(\phi_{I}(s)+i \psi_{I}(s)\right)\left[\phi_{I}^{\prime}(s)+i \psi_{I}^{\prime}(s)\right]$, converges for any path $\xi=\phi_{I}(s), \eta=\psi_{I}(s)$ that envelopes $\Omega_{\Gamma_{S}}$. Then the DunfordCauchy integral

$F(\mathcal{L})=\frac{1}{2 \pi i} \int_{\Gamma_{I}} F(z)(z I-\mathcal{L})^{-1} d z=-\frac{1}{2 \pi i} \int_{-\infty}^{\infty} \Phi_{1}(s)\left[\left(\phi_{I}(s)+i \psi_{I}(s)\right) I-\mathcal{L}\right]^{-1} d s$ defines a bounded operator $F(\mathcal{L})$.

Proof. Estimate (2.6) for the resolvent and the assumptions above imply the bound

$$
\|F(\mathcal{L})\| \leq \int_{-\infty}^{\infty}\left|\Phi_{1}(s) f_{R}\left(\phi_{I}(s)+i \psi_{I}(s)\right)\right| d s<\infty .
$$

We further consider examples of quadrature rules applied to the integrals in Theorem 2.2 Our particular application is concerned with the operator exponential $\mathcal{F}_{1}(\mathcal{L})$ and with the solution operator $\mathcal{F}_{4}(\mathcal{L})$ (see (1.1)) to the Lyapunov equation arising in control theory.

\subsection{Representation of the operator exponential by resolvents.}

2.4.1. Dunford-Cauchy integral. In this section, we briefly recall (and slightly modify) the results from [7]. In order to get exponentially convergent discretisations, we are interested in operators of $\left(f_{S}, f_{R}\right)$-type with an exponentially decreasing function $\left|\Phi_{1}(s) f_{R}\left(\phi_{I}(s)+i \psi_{I}(s)\right)\right|$. The rate of decay obviously depends on the functions $\phi_{I}(s), \psi_{I}(s), \phi_{S}(s), \psi_{S}(s)$. Let $\mathcal{L}$ be a linear, densely defined, closed operator of $\left(f_{S}, f_{R}\right)$-type in a Banach space $X$, where

$$
\begin{aligned}
& \xi=\phi_{S}(s)=a_{p} s^{2}+b_{p}, \\
& \eta=\psi_{S}(s)=s .
\end{aligned}
$$

The function $f_{S}(\eta)$ defines the so-called spectral curve

$$
\Gamma_{S}=\left\{z=\xi+i \eta: \xi=a_{p} \eta^{2}+b_{p}\right\},
$$


containing the spectrum $s p(\mathcal{L})$ of the operator $\mathcal{L}$. We choose the integration curve $\Gamma_{I}$ as the hyperbola

$$
\begin{aligned}
& \xi=\phi_{I}(s)=a_{e} \cosh s, a_{e}<b_{p}, \\
& \eta=\psi_{I}(s)=b_{e} \sinh s .
\end{aligned}
$$

The equation of this hyperbola in the coordinates $\xi, \eta$ is

$$
\frac{\xi^{2}}{a_{e}^{2}}-\frac{\eta^{2}}{b_{e}^{2}}=1
$$

This hyperbola belongs to the family of hyperbolas given by

$$
\begin{aligned}
& \xi=\phi_{F}(s, \nu)=a_{F}(\nu) \cosh s, \\
& \eta=\psi_{F}(s, \nu)=b_{F}(\nu) \sinh s,
\end{aligned}
$$

where

$$
\begin{aligned}
& a_{F}(\nu)=\sqrt{a_{e}^{2}+b_{e}^{2}} \cos (\nu+\varphi), b_{F}(\nu)=\sqrt{a_{e}^{2}+b_{e}^{2}} \sin (\nu+\varphi), \\
& \cos \varphi=\frac{a_{e}}{\sqrt{a_{e}^{2}+b_{e}^{2}}}, \sin \varphi=\frac{b_{e}}{\sqrt{a_{e}^{2}+b_{e}^{2}}} .
\end{aligned}
$$

Setting $\nu=0$ here, we obtain the integration hyperbola (2.10). We choose the parameter $\nu=-d$ so that the hyperbola $\Gamma_{I S}$ defined by $\xi=\phi_{F}(s,-d), \quad \eta=$ $\psi_{F}(s,-d)$ with some $d>0$, envelopes the spectral parabola.

In order to estimate the distance $d_{I S}$ between the spectral and the integration curves, we note that for a fixed $s$ the line

$$
\left(\begin{array}{l}
\xi \\
\eta
\end{array}\right)=\left(\begin{array}{c}
a_{p} s^{2}+b_{p} \\
s
\end{array}\right)+\lambda\left(\begin{array}{c}
-1 \\
2 a_{p} s
\end{array}\right), \quad \lambda \in(-\infty, \infty),
$$

in the coordinate plane $\xi, \eta$ is orthogonal to the spectral curve at the point defined by this $s$. The system of equations

$$
\left(\begin{array}{c}
a_{e} \cosh s \\
b_{e} \sinh s
\end{array}\right)=\left(\begin{array}{c}
a_{p} s^{2}+b_{p} \\
s
\end{array}\right)+\lambda\left(\begin{array}{c}
-1 \\
2 a_{p} s
\end{array}\right), \quad \lambda \in(-\infty, \infty)
$$

defines $s, \lambda$ for which the normal line to the spectral curve intersects the integration curve. These equations imply

$$
|\lambda| \sqrt{1+4 a_{p}^{2} s^{2}}=\sqrt{\left(a_{e} \cosh s-a_{p} s^{2}-b_{p}\right)^{2}+\left(b_{e} \sinh s-s\right)^{2}}
$$

from where $\lambda \asymp|s|^{-1} e^{|s|}$ for $|s|$ large enough. Now, it follows from (2.15) that the distance $d_{I S}$ possesses the asymptotical behaviour $d_{I S} \asymp e^{|s|}$; therefore the resolvent can be estimated on the integration curve by

$$
\left\|(z I-\mathcal{L})^{-1}\right\| \leq M e^{-|s|}
$$

with some positive constant $M$ for $|s|$ large enough.

In the following, we use the infinite strip

$$
D_{d}:=\{z \in \mathbb{C}:-\infty<\Re e z<\infty,|\Im m z|<d\}
$$

as well as the finite rectangles $D_{d}(\epsilon)$ defined for $0<\epsilon<1$ by

$$
D_{d}(\epsilon)=\{z \in \mathbb{C}:|\Re e z|<1 / \epsilon,|\Im m z|<d(1-\epsilon)\} .
$$


For $1 \leq p \leq \infty$, introduce the space $\mathbf{H}^{p}\left(D_{d}\right)$ of all operator-valued functions which are analytic in $D_{d}$, such that for each $\mathcal{F} \in \mathbf{H}^{p}\left(D_{d}\right)$ it holds that $\|\mathcal{F}\|_{\mathbf{H}^{p}\left(D_{d}\right)}<\infty$ with

$$
\|\mathcal{F}\|_{\mathbf{H}^{p}\left(D_{d}\right)}:= \begin{cases}\lim _{\epsilon \rightarrow 0}\left(\int_{\partial D_{d}(\epsilon)}\|\mathcal{F}(z)\|^{p}|d z|\right)^{1 / p} & \text { if } 1 \leq p<\infty \\ \lim _{\epsilon \rightarrow 0} \sup _{z \in D_{d}(\epsilon)}\|\mathcal{F}(z)\| & \text { if } p=\infty .\end{cases}
$$

The next lemma justifies an integral representation for $e^{-t \mathcal{L}}$ generated by an $\left(f_{S}, f_{R}\right)$-type operator $\mathcal{L}$.

Lemma 2.3. Let $\mathcal{L}$ be an $\left(f_{S}, f_{R}\right)$-type operator with the spectral curve $\Gamma_{S}$ defined by (2.9). Choose the (integration) curve $\Gamma_{I}=\left\{z=\xi+i \eta: \xi=a_{e} \cosh (s), \eta=\right.$ $\left.b_{e} \sinh s\right\}$ with $a_{e}, b_{e}$ such that $\Gamma_{I}$ envelops $\Gamma_{S}$. Then the operator exponential $I(t ; \mathcal{L})=e^{-t \mathcal{L}}$ can be represented by the Dunford-Cauchy integral

$$
I(t ; \mathcal{L})=\frac{1}{2 \pi i} \int_{\Gamma_{I}} e^{-z t}(z I-\mathcal{L})^{-1} d z=-\frac{1}{2 \pi i} \int_{-\infty}^{\infty} F_{1}(s, t) d s,
$$

where the integrand

$$
\begin{aligned}
& F_{1}(s, t)=e^{-z t} z^{\prime}(s)(z I-\mathcal{L})^{-1}, \\
& z=a_{e} \cosh (s)+i b_{e} \sinh (s), z^{\prime}(s)=a_{e} \sinh (s)+i b_{e} \cosh (s), s \in \mathbb{R},
\end{aligned}
$$

can be estimated on the real axis by

$$
\left\|F_{1}(\eta, t)\right\| \leq M_{1} e^{-t \sqrt{a_{e}^{2}+b_{e}^{2}}|\sinh s|} \quad \text { for } s \in \mathbb{R}
$$

with some positive constant $M_{1}$. Moreover, $F_{1}(\cdot, t)$ can be analytically extended into the strip $D_{d}$ of the width $d>0$ and belongs to the class $\mathbf{H}^{p}\left(D_{d}\right)$ for all $p \in[1, \infty]$.

Proof. The estimate (2.22) follows immediately from (2.17). In order to show that the integrand can be extended analytically into a strip, we substitute $s=\mu+i \nu$ into (2.21). The analyticity can obviously be violated only if the set $\{z=\xi+i \eta$ : $\left.\xi=a_{e} \cosh (\mu+i \nu), \eta=b_{e} \sinh (\mu+i \nu), \mu \in(-\infty, \infty),|\nu|<d\right\}$ intersects the spectral curve in the plane $\xi, \eta$ (in this case the resolvent becomes unbounded). For each $\nu$ we consider this set as a curve in the variable $\mu$. We have

$$
\begin{aligned}
z & =a_{e} \cosh (\mu+i \nu)+i b_{e} \sinh (\mu+i \nu) \\
& =a_{e} \cosh \mu \cos \nu+i a_{e} \sinh \mu \sin \nu+i b_{e}(\sinh \mu \cos \nu+i \cosh \mu \sin \nu) \\
& =\cosh \mu\left(a_{e} \cos \nu-b_{e} \sin \nu\right)+i \sinh \mu\left(a_{e} \sin \nu+b_{e} \cos \nu\right) \\
& =\sqrt{a_{e}^{2}+b_{e}^{2}}[\cos (\nu+\varphi) \cosh \mu+i \sin (\nu+\varphi) \sinh \mu] \\
& =\sqrt{a_{e}^{2}+b_{e}^{2}} \cosh [\mu+i(\nu+\varphi)],
\end{aligned}
$$

where $\phi$ is such that

$$
\cos \varphi=\frac{a_{e}}{\sqrt{a_{e}^{2}+b_{e}^{2}}}, \quad \sin \varphi=\frac{b_{e}}{\sqrt{a_{e}^{2}+b_{e}^{2}}} .
$$

For each $\nu$, the set (2.23) is the following hyperbola (parametrised by $\mu$ ):

$$
\begin{aligned}
& \xi=\sqrt{a_{e}^{2}+b_{e}^{2}} \cos (\nu+\varphi) \cosh \mu, \\
& \eta=\sqrt{a_{e}^{2}+b_{e}^{2}} \sin (\nu+\varphi) \sinh \mu .
\end{aligned}
$$

Since the function $\cos (\nu+\varphi)$ decreases and the function $\sin (\nu+\varphi)$ increases monotonically for $\nu \in(-\varphi, \pi / 2-\varphi)$, we see that for $|\nu|<d$ with $d=\min \{\varphi, \pi / 2-\varphi\}$ 


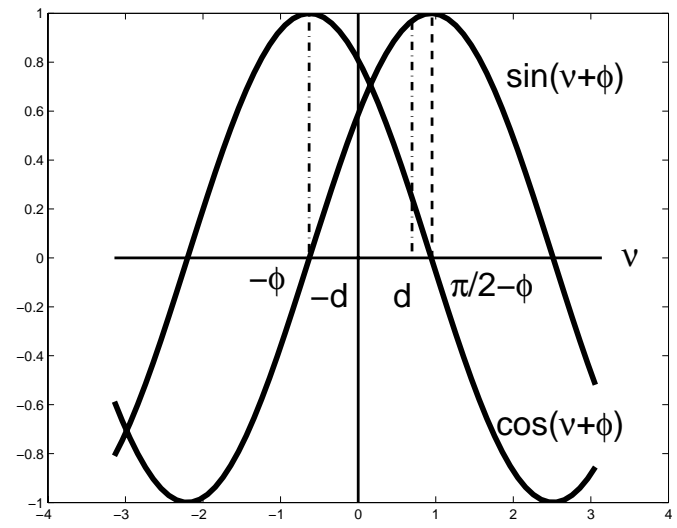

Figure 2. Calculation of the width of the analyticity strip.

and for all $\mu \in(-\infty, \infty)$ the set of hyperbolas still lies in the right half-plane and envelopes the hyperbola $\Gamma_{I S}$ and spectral parabola without intersecting them; i.e., we can analytically extend the integrand into the strip of the width $2 d$ (see also Figure 2). The proof is complete

We denote the parametric set of hyperbolas (2.25) with $|\nu|<d$ by $H_{I}$. The class of the $\left(f_{S}, f_{R}\right)$-type operators with the spectral parabola which can be enveloped by $H_{I}$ is called $\mathbf{E}_{H}$.

2.4.2. Sinc quadrature applied to the exponential. Following [29] 9, 7], we construct a quadrature rule for the integral in 2.20) by using the Sinc approximation. Let

$$
S(k, h)(x)=\frac{\sin [\pi(x-k h) / h]}{\pi(x-k h) / h} \quad(k \in \mathbb{Z}, h>0, x \in \mathbb{R})
$$

be the $k$-th Sinc function with step size $h$, evaluated at $x$. Given $f \in \mathbf{H}^{p}\left(D_{d}\right), h>0$, and $N \in \mathbb{N}$, we use the notation

$$
\begin{array}{rlrl}
I(f) & =\int_{\mathbb{R}} f(\xi) d \xi & \\
T(f, h) & =h \sum_{k=-\infty}^{\infty} f(k h), & T_{N}(f, h)=h \sum_{k=-N}^{N} f(k h), \\
C(f, h) & =\sum_{k=-\infty}^{\infty} f(k h) S(k, h), & E(f, h)=f-C(f, h), \\
\eta(f, h) & =I(f)-T(f, h), & & \eta_{N}(f, h)=I(f)-T_{N}(f, h)
\end{array}
$$

( $I$ : integral; $T$ : trapezoidal rule, $\eta, \eta_{N}$ : quadrature errors). Further, we need the notation of one-sided limits:

$$
f\left(\xi \pm i d^{-}\right)=\lim _{\delta \rightarrow d ; \delta<d} f(\xi \pm i \delta) \quad \text { for } \xi, d \in \mathbb{R} .
$$

The following approximation results for functions from $\mathbf{H}^{1}\left(D_{d}\right)$ describe the accuracy of $T_{N}(f, h)$ (cf. [7], also for the proof of Lemma [2.4 below). 
Lemma 2.4. For any operator-valued function $f \in \mathbf{H}^{1}\left(D_{d}\right)$ satisfying on $\mathbb{R}$ the condition

$$
\|f(\xi)\|<c \exp (-b|\sinh \xi|), \quad b, c>0
$$

it holds that

$$
\left\|\eta_{N}(f, h)\right\| \leq C\left[\frac{e^{-2 \pi d / h}}{1-e^{-2 \pi d / h}}\|f\|_{\mathbf{H}^{1}\left(D_{d}\right)}+\frac{1}{b} \exp (-b \sinh (h N))\right]
$$

with the constant d from $\mathbf{H}^{1}\left(D_{d}\right)$.

Proof. Let $E(f, h)$ be the error from (2.28). Analogously to [29, Thm. 3.1.2], $E(f, h)(z)$ equals

$$
\frac{\sin (\pi z / h)}{2 \pi i} \int_{\mathbb{R}}\left\{\frac{f(\xi-i d)}{(\xi-z-i d) \sin [\pi(\xi-i d) / h]}-\frac{f(\xi+i d)}{(\xi-z+i d) \sin [\pi(\xi+i d) / h]}\right\} d \xi,
$$

and after replacing $z$ by $x$, we have

$$
\eta(f, h)=\int_{\mathbb{R}} E(f, h)(x) d x
$$

because of $\int_{\mathbb{R}} S(k, h)(x) d x=h$. After interchanging the order of integration and using

$$
\frac{1}{2 \pi i} \int_{\mathbb{R}} \frac{\sin (\pi x / h)}{ \pm(\xi-x)-i d} d x=\frac{i}{2} e^{-\pi(d \pm i \xi) / h},
$$

we obtain

$$
\eta(f, h)=\frac{i}{2} \int_{\mathbb{R}}\left\{\frac{f\left(\xi-i d^{-}\right) e^{-\pi(d+i \xi) / h}}{\sin [\pi(\xi-i d) / h]}-\frac{f\left(\xi+i d^{-}\right) e^{-\pi(d-i \xi) / h}}{\sin [\pi(\xi+d) / h]}\right\} d \xi .
$$

Using the estimate $\sinh (\pi d / h) \leq|\sin [\pi(\xi \pm i d) / h]| \leq \cosh (\pi d / h)$ (see [29, p. 133]), the assumption $f \in \mathbf{H}^{1}\left(D_{d}\right)$ and identity (2.31), we obtain the bound

$$
\|\eta(f, h)\| \leq \frac{e^{-\pi d / h}}{2 \sinh (\pi d / h)}\|f\|_{\mathbf{H}^{1}\left(D_{d}\right)}
$$

The assumption (2.29) now implies

$$
\begin{aligned}
\left\|\eta_{N}(f, h)\right\| & \leq\|\eta(f, h)\|+h \sum_{|k|>N}\|f(k h)\| \\
& \leq \frac{\exp (-\pi d / h)}{2 \sinh (\pi d / h)}\|f\|_{\mathbf{H}^{1}\left(D_{d}\right)}+c h \sum_{k:|k|>N} \exp (-b|\sinh (k h)|) .
\end{aligned}
$$

For the last sum we use the simple estimate

$$
\begin{aligned}
& \sum_{k:|k|>N} \exp (-b|\sinh (k h)|)=2 \sum_{k=N+1}^{\infty} \exp (-b|\sinh (k h)|) \\
& \leq 2 \int_{N}^{\infty} \exp (-b|\sinh (x h)|) d x \\
& \leq 2 \int_{N}^{\infty} \cosh (x h) \exp (-b|\sinh (x h)|) d x=\frac{2}{b h} \exp (-b \sinh (h N)),
\end{aligned}
$$

which together with (2.33) and (2.35) implies (2.30). 
The operator exponential $I(t ; \mathcal{L})=e^{-t \mathcal{L}}$ is represented as integral according to Lemma 2.3. Applying the quadrature rule $T_{N}$ to the operator-valued function $f(\eta):=-\frac{1}{2 \pi i} F_{1}(\eta, t)$, where $F_{1}(\eta, t)$ is given by (2.21), we obtain for the operator family $\{I(t) \equiv I(t ; \mathcal{L}): t>0\}($ cf. $(2.26))$ that

$$
I(t) \approx T_{N}(t) \equiv T_{N}(f, h)=-\frac{h}{2 \pi i} \sum_{k=-N}^{N} F_{1}(k h, t) .
$$

The error analysis is given by the following theorem.

Theorem 2.5. Given the spectral curve $\Gamma_{S}$ from (2.9) associated with $f_{S}(\eta)=$ $a_{p} \eta^{2}+b_{p}$, choose the integration curve $\left\{z=\xi+i \eta: \xi=a_{e} \cosh s, \eta=b_{e} \sinh s, s \in\right.$ $\mathbb{R}\}$ and set $h=\frac{\log N}{N}$. Then

$$
\left\|I(t)-T_{N}(t)\right\| \lesssim \frac{1}{t \sqrt{a_{e}^{2}+b_{e}^{2}}}\left(e^{-2 \pi d N / \log N}+e^{-t \sqrt{a_{e}^{2}+b_{e}^{2}} N}\right) .
$$

Proof. Substituting in (2.30) $F_{1}$ for $f, t \sqrt{a_{e}^{2}+b_{e}^{2}}$ for $b$ and specifying $h=\frac{\log N}{N}$, we conclude (2.37) from the bound $\|f\|_{\mathbf{H}^{1}\left(D_{d}\right)} \leq C b^{-1}$.

The exponential convergence of our quadrature rule allows us to introduce the following algorithm for the approximation of the operator exponential with a given tolerance $\varepsilon>0$. Note that the time-variable $t \in(0, \infty)$ enters only the coefficients of our quadrature rule, while all resolvents appear to be independent on $t$.

Proposition 2.6. (a) Let $\varepsilon>0$ be given. In order to obtain $\left\|I(t)-T_{N}(t)\right\| \lesssim \frac{\varepsilon}{t}$ uniformly with respect to $t>0$, choose

$$
\begin{aligned}
N & =O(|\log \varepsilon|), \quad h=\frac{\log N}{N}, \\
z_{k} & =z(k h)=a_{e} \cosh (k h)+i b_{e} \sinh (k h), z^{\prime}(k h)=a_{e} \sinh (k h)+i b_{e} \cosh (k h), \\
\gamma_{k}(t) & =e^{-z(k h) t} \frac{h}{2 \pi i} z^{\prime}(k h),(k=-N, \ldots, N) .
\end{aligned}
$$

Then $T_{N}(t)$ is a linear combination of $2 N+1$ resolvents with scalar weights depending on $t$ :

$$
T_{N}(t)=\sum_{k=-N}^{N} \gamma_{k}(t)\left(z_{k} I-\mathcal{L}\right)^{-1}
$$

so that the computation of $T_{N}(t)$ requires $2 N+1=O(|\log \varepsilon|)$ evaluations of the resolvents $\left(z_{k} I-\mathcal{L}\right)^{-1}, k=-N, \ldots, N$.

(b) The evaluations (or approximations) of the resolvents can be performed in parallel. Note that the shifts $z_{k}$ are independent of $t$.

(c) Having evaluated the resolvents, $T_{N}(t)$ can be determined in parallel for different $t$-values $t_{1}, t_{2}, \ldots$.

Proof. Use (2.37) for the error estimate.

2.4.3. Exponentially convergent algorithm for the operator exponential with $t \geq 0$. The algorithm (2.36) does not provide the exponential accuracy for $t \rightarrow 0$. In this section we show that a slightly modified algorithm for the weighted operator 
exponential $T_{\sigma}(t)=\mathcal{L}^{-\sigma} e^{-t \mathcal{L}}, t \geq 0, \sigma>1$, guarantees the exponential convergence rate for all $t \geq 0$. Applied to the initial-value parabolic problem

$$
\frac{d u}{d t}+A u=0, \quad u(0)=u_{0},
$$

we can get $u(t)=T_{\sigma}(t) u_{0, \sigma}$ with $u_{0, \sigma}=\mathcal{L}^{\sigma} u_{0}$ provided that $u_{0} \in D\left(\mathcal{L}^{\sigma}\right)$; i.e., in this case we need sufficient regularity of initial data. Choose a curve (integration curve) $\Gamma_{I}=\left\{z=\xi+i \eta: \xi=a_{e} \cosh s, \eta=\sinh s\right\}$ as before. Then the weighted operator exponential $T_{\sigma}(t)=\mathcal{L}^{-\sigma} e^{-t \mathcal{L}}$ can be represented by the Dunford-Cauchy integral

$$
T_{\sigma}(t)=\frac{1}{2 \pi i} \int_{\Gamma_{I}} z^{-\sigma} e^{-z t}(z I-\mathcal{L})^{-1} d z=-\frac{1}{2 \pi i} \int_{-\infty}^{\infty} F_{1, \sigma}(\eta, t) d s,
$$

where the integrand

$$
F_{1, \sigma}(s, t)=z^{-\sigma} e^{-z t} z^{\prime}(s)(z I-\mathcal{L})^{-1}
$$

with $z(s)=a_{e} \cosh s+i b_{e} \sinh s, s \in \mathbb{R}$. Contrary to the result of Lemma 2.3, the function $F_{1, \sigma}(s, t)$ can be estimated on the real axis by

$$
\begin{aligned}
\left\|F_{1, \sigma}(\eta, t)\right\| & \lesssim e^{-t \sqrt{a_{e}^{2} \cosh ^{2} s+b_{e}^{2} \sinh ^{2} s}}\left(a_{e}^{2} \cosh ^{2} s+b_{e}^{2} \sinh ^{2} s\right)^{-\sigma / 2} \\
& \lesssim e^{-\sigma|s|-t \sqrt{a_{e}^{2}+b_{e}^{2}}|\sinh s|} \lesssim e^{-\sigma|s|} \quad \text { for } s \in \mathbb{R},
\end{aligned}
$$

for all $t \geq 0$. Moreover, $F_{1}(\cdot, t)$ can be analytically extended into the strip $D_{d}$ of the width $d>0$ constructed above and belongs to the class $\mathbf{H}^{p}\left(D_{d}\right)$ for all $p \in[1, \infty]$. Applying the quadrature rule (2.27)

$$
T_{\sigma}(t) \approx T_{\sigma, N}(t)=h \sum_{k=-N}^{N} F_{1, \sigma}(k h, t)
$$

to the function $f(t)=F_{1, \sigma}(s, t)$, we can bound the error $\eta_{N}(f, h)$ (see [29, [5]) by

$$
\left\|\eta_{N}\right\|=\left\|T_{\sigma}(t)-T_{\sigma, N}(t)\right\| \leq c\left[\frac{e^{-\pi d / h}}{2 \sinh \pi d / h}+(\sigma)^{-1} e^{-\sigma N h}\right]\left\|F_{1, \sigma}\right\|_{\mathbf{H}^{1}\left(D_{d}\right)} .
$$

Equalising the exponents by setting $h=\sqrt{\pi d /(\sigma N)}$, we get the error estimate

$$
\left\|\eta_{N}\right\| \leq c e^{-\sqrt{\pi d \sigma N}}\left\|F_{1, \sigma}\right\|_{\mathbf{H}^{1}\left(D_{d}\right)} \quad \forall t \geq 0 .
$$

Thus, we can prove the following

Proposition 2.7. (a) Let $\varepsilon>0$ be given. In order to obtain $\left\|T_{\sigma}(t)-T_{\sigma, N}(t)\right\| \lesssim \varepsilon$ uniformly with respect to $t \geq 0$, choose

$$
\begin{aligned}
N & =O(|\log \varepsilon|), \quad h=\sqrt{\pi d /[\sigma N]}, \\
z_{k} & =z(k h)=\xi(k h)+i \psi(k h)(k=-N, \ldots, N), \\
\xi(s) & =a_{e} \cosh s, \psi(s)=b_{e} \sinh s, \\
\gamma_{\sigma, k}(t) & =z_{k}^{-\sigma} e^{-z_{k} t} \frac{h}{2 \pi i} z^{\prime}(k h) .
\end{aligned}
$$

Then $T_{\sigma, N}(t)$ is a linear combination of $2 N+1$ resolvents with scalar weights depending on $t$ :

$$
T_{\sigma, N}(t)=\sum_{k=-N}^{N} \gamma_{\sigma, k}(t)\left(z_{k} I-\mathcal{L}\right)^{-1},
$$


so that the computation of $T_{\sigma, N}(t)$ requires $2 N+1=O\left(|\log \varepsilon|^{2}\right)$ evaluations of the resolvents $\left(z_{k} I-\mathcal{L}\right)^{-1}, k=-N, \ldots, N$.

(b) The evaluations (or approximations) of the resolvents can be performed in parallel. Note that the shifts $z_{k}$ are independent of $t$.

(c) Having evaluated the resolvents, $T_{\sigma, N}(t)$ can be determined in parallel for different $t$-values $t_{1}, t_{2}, \ldots$.

In practice one prefers integers $\sigma$, so that $\sigma=2$ is the first choice. To conclude this section, we note that the condition $u_{0} \in D\left(\mathcal{L}^{\sigma}\right)$ is no longer an essential restriction if $\mathcal{L}$ is a finite-dimensional operator (say, the discrete elliptic operator). We refer to Remark 4.3 for further results concerning the matrix exponential.

2.5. Operator-valued functions in control theory. In this section we recall the results in [7. Let us consider the linear dynamical system of equations

$$
\frac{d X(t)}{d t}=A X(t)+X(t) B+G, \quad X(0)=X_{0}
$$

where $X(t), A, B, G \in \mathbb{R}^{n \times n}(A, B, G$ given constant matrices). The solution $X(t)$ is given by

$$
X(t)=e^{t A} X_{0} e^{t B}+\int_{0}^{t} e^{(t-s) A} G e^{(t-s) B} d s .
$$

If all eigenvalues of $A, B$ have negative real parts, then the limit

$$
X(t) \rightarrow X_{\infty}=\int_{0}^{\infty} e^{t A} G e^{t B} d t \quad(t \rightarrow \infty)
$$

exists and the $X_{\infty}$ satisfies the matrix Lyapunov-Sylvester equation

$$
A X_{\infty}+X_{\infty} B+G=0 .
$$

We refer to 3 . concerning the proof of (2.47) in the case of a matrix equation. The operator case considered below can be treated similarly.

2.5.1. Integral operator-to-operator mapping. We set $A=\mathcal{L}^{*}, B=\mathcal{L}$ in (2.48), where $\mathcal{L}: V \rightarrow V^{\prime}$ is an elliptic second order differential operator, and we consider the solution of the operator Lyapunov equation: Find a selfadjoint continuous operator $\mathcal{Z}: L^{2}(\Omega) \rightarrow V$ such that

$$
\mathcal{L}^{*} \mathcal{Z}+\mathcal{Z} \mathcal{L}+G=0 .
$$

The solution $\mathcal{Z}$ of the operator Lyapunov equation is given by

$$
\mathcal{Z}(\mathcal{L}):=\int_{0}^{\infty} \mathcal{G}_{0}(t, \mathcal{L}, G) d t
$$

where $\mathcal{G}_{0}(z, \mathcal{L}, G):=e^{z \mathcal{L}^{*}} G e^{z \mathcal{L}}$ is a continuous operator-valued function of $z \in$ $[0, \infty)$, of an elliptic second order operator $\mathcal{L}$ and of a selfadjoint operator $G$ : $L^{2}(\Omega) \rightarrow L^{2}(\Omega)$ (see [3] for the matrix case).

In this section we consider a class of operator-valued functions defined by an integral representation $(2.50)$ on $\Gamma:=[0, \infty)$. The operator-valued function $\mathcal{Z}(\mathcal{L})$ defines the solution operator to the Lyapunov equation (2.49) arising in control theory. As usual in control theory, we further assume $\Re$ e $\operatorname{sp}(-\mathcal{L}) \subset\left(\lambda_{0}, \infty\right)$, with $\lambda_{0}>0$. 


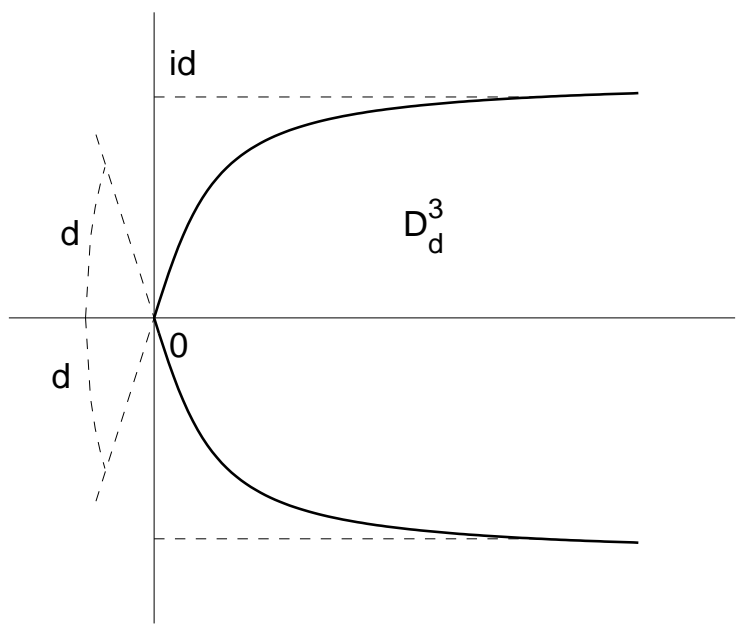

FiguRE 3 . The region $\mathcal{D}_{d}$

To simplify the discussion, we assume that $-\mathcal{L}$ is the elliptic operator of the $\left(f_{S}, f_{R}\right)$-type with $f_{S}, f_{R}$ given by (2.7) such that

$$
s p(-\mathcal{L}) \subset S_{\mu}:=\left\{z \in \mathbb{C}:|\Im m z| \leq \mu, \quad \Re e z>\lambda_{0}\right\}, \quad \lambda_{0}, \mu>0 .
$$

In particular, the latter condition implies that the elliptic operator $\mathcal{L}$ generates a strongly continuous semi-group $e^{t \mathcal{L}}$ such that

$$
\left\|e^{t \mathcal{L}}\right\| \leq C \frac{1+\mu^{2}}{\lambda_{0} \sqrt{t}} e^{-c \lambda_{0} t} \quad \text { for all } t \in[0, \infty) \quad(\|\cdot\|: \text { spectral operator norm }),
$$

where $C, c>0$ do not depend on $\lambda_{0}$ and $t$.

In the following we discuss exponentially convergent quadrature rules for the integral (2.50). The construction consists of two steps: First, we approximate the integral by a sum of operators $\mathcal{G}_{0}\left(t_{k}, \mathcal{L}, G\right)$ computed in a few quadrature points $t_{k} \in$ $[0, \infty)$, and then we approximate each operator exponential involved in $\mathcal{G}_{0}\left(t_{k}, \mathcal{L}, G\right)$ as in $\S 2.4$. The resultant quadrature rule is similar to that in [11, Thm. 5] for the case of a matrix Lyapunov equation. Contrary to [11, here we consider the integral of an operator-valued function; moreover, we apply the more efficient approximation scheme of $\S 2.4$.

Applying the substitution $t=u^{\alpha}$ in the integral of (2.50) for some $\alpha \geq 0$, we obtain the equivalent representation

$$
\mathcal{Z}(\mathcal{L})=\int_{0}^{\infty} \mathcal{G}_{\alpha}(t, \mathcal{L}, G) d t \quad \text { with } \mathcal{G}_{\alpha}(t, \mathcal{L}, G):=g_{\alpha}\left(t, \mathcal{L}^{*}\right) G g_{\alpha}(t, \mathcal{L}) d t
$$

where

$$
g_{\alpha}(z, \lambda):=\sqrt{1+2 \alpha} z^{\alpha} e^{z^{1+2 \alpha} \lambda}, \quad \alpha \geq 0,
$$

so that (2.50) corresponds to the case $\alpha=0$.

First, we recall some auxiliary approximation results for holomorphic functions based on the Sinc approximation. Let the region $1 \mathcal{D}_{d}$ (see Figure 3i) for a given

\footnotetext{
${ }^{1}$ This is the domain called $\mathcal{D}_{d}^{3}$ in [29]. Note that it is different from the strip $D_{d}$ in (2.18).
} 
$d \in(0, \pi / 2]$ be defined by

$$
\mathcal{D}_{d}:=\{z \in \mathbb{C}:|\arg (\sinh z)|<d\} .
$$

We denote by $H^{1}\left(\mathcal{D}_{d}\right)$ the family of functions that are analytic in $\mathcal{D}_{d}$ and satisfy

$$
N_{1}\left(f, \mathcal{D}_{d}\right):=\int_{\partial \mathcal{D}_{d}}|f(z)||d z|<\infty .
$$

Now, for $\alpha, \beta \in(0,1]$, introduce the space

$$
L_{\alpha, \beta}\left(\mathcal{D}_{d}\right):=\left\{f \in H^{1}\left(\mathcal{D}_{d}\right):|f(z)| \leq C\left(\frac{|z|}{1+|z|}\right)^{\alpha} e^{-\beta \Re e z} \quad \text { for all } z \in \mathcal{D}_{d}\right\}
$$

(cf. [29]). We set

$$
\phi(z):=\log \{\sinh (z)\},
$$

hence

$$
\phi^{\prime}(z)=\frac{1}{\tanh (z)}, \quad z=\phi^{-1}(w)=\log \left(e^{w}+\sqrt{1+e^{2 w}}\right),
$$

where $\phi(z)$ is the conformal map of $\mathcal{D}_{d}$ onto the infinite strip $D_{d}$ defined by (2.18).

It is easy to check that

$$
g_{\alpha}(\cdot, \lambda) \in L_{\alpha, \beta}, \quad \beta \geq \min \left\{1, a_{0}\right\} \quad \text { for } \lambda \in \Gamma_{\mathcal{L}},
$$

where, with given $a, b_{0}>0, \Gamma_{\mathcal{L}}:=\left\{z=\xi+i \eta: \xi=-b_{0}-a \eta^{2}, \eta \in(-\infty, \infty)\right\}$ is the integration parabola to be used for technical needs.

2.5.2. Quadrature Rule I applied to $\mathcal{Z}(\mathcal{L})$. In the sequel, the value of $d$ involved in $\mathcal{D}_{d}$ (cf. (2.54) ) will be chosen from the interval $0<d<\frac{\pi}{2(1+2 \alpha)}$, where we further fix the parameter $\alpha=1 / 2$. Without loss of generality we can assume $\beta=\min \left\{1, \lambda_{0} / 2\right\} \leq \alpha=1 / 2$ and then choose the parameter $b_{0}$ defining the integrating parabola $\Gamma_{\mathcal{L}}$, by $b_{0}=\lambda_{0} / 2$. For ease of notation we further omit the subscript $\alpha$ in $g_{\alpha}$ and set $g=g_{1 / 2}$.

Lemma 2.8. Let the spectrum of $\mathcal{L}$ lie in the strip $S_{\mu}$ defined by (2.51). Given $N \in \mathbb{N}$ and $\beta=\min \left\{1, \lambda_{0} / 2\right\}$, with the proper choice of $h>0, t_{k} \in[0, \infty)$, and $M$ given by

$$
h=\sqrt[3]{\frac{\pi d}{2 \lambda_{0} N^{2}}}, t_{k}=\log \left(e^{k h}+\sqrt{1+e^{2 k h}}\right), M=\lceil 2 \beta N\rceil,
$$

the integral $\mathcal{Z}(\mathcal{L})=\int_{0}^{\infty} \mathcal{G}_{0}(t, \mathcal{L}, G) d t$ allows an exponentially convergent quadrature rule

$$
\mathcal{Z}_{N}(\mathcal{L}):=h \sum_{k=-M}^{N} \tanh \left(t_{k}\right) \mathcal{G}_{1 / 2}\left(t_{k}, \mathcal{L}, G\right),
$$

providing the error estimate

$$
\left\|\mathcal{Z}(\mathcal{L})-\mathcal{Z}_{N}(\mathcal{L})\right\| \leq C\left(1+\mu^{2}\right) e^{-\left(2 \pi \lambda_{0} d N\right)^{2 / 3}}
$$

with constants $C$ independent of $N$ and with $\mu$ being half the width of the strip $S_{\mu}$ in (2.51).

Proof. See [7]. 
Lemma 2.8 already provides a low rank approximation to the solution of the Lyapunov equation in the case when a right-hand side $G$ has this property. In fact, let us assume that $G$ has a separable representation consisting of $k_{G}$ terms, i.e.,

$$
G:=\sum_{j=1}^{k_{G}} a_{j} * f_{j}
$$

where $f_{j}: L^{2}(\Omega) \rightarrow \mathbb{R}$ are linear continuous functionals, while $a_{j} \in L^{2}(\Omega)$ are functions on $\Omega$. Substitution of the above representation into $\mathcal{G}_{1 / 2}\left(t_{k}, \mathcal{L}, G\right)$ shows that $\mathcal{G}_{1 / 2}\left(t_{k}, \mathcal{L}, G\right)$ is also separable with $k_{G}$ terms. Due to $2.58, \mathcal{Z}_{N}(\mathcal{L})$ is separable with $k=k_{G}(N+M+1)$ terms (in the matrix case, this is equivalent to $\left.\operatorname{rank} \mathcal{Z}_{N}(\mathcal{L}) \leq k\right)$

We proceed with the approximation of the individual terms $\mathcal{G}_{1 / 2}\left(t_{k}, \mathcal{L}, G\right)$ in (2.58). For this purpose, we apply the basic construction from $\S 2.4$ modified by a proper translation transform explained below. We use the symbol $A$ for both $\mathcal{L}$ and $\mathcal{L}^{\top}$. We recall that with a given elliptic operator $A$ and for the described choice of the parameters $z_{p}, h, c_{p}$, the quadrature

$$
\exp _{L}(A)=\sum_{p=-L}^{L} c_{p} e^{-z_{p}}\left(z_{p} I-A\right)^{-1} \quad(\text { see }(2.38))
$$

provides exponential convergence (cf. (2.37)). To adapt the above approximation to our particular situation, we include the parameter $t_{k}$ into the operator by setting $A_{k}:=t_{k}^{2} \mathcal{L}$, which then leads to the bound $\lambda_{\min }\left(A_{k}\right)=O\left(t_{k}^{2}\right)$. Due to the factor $\frac{1}{t}$ in (2.37), the error estimate deteriorates when $t_{k} \rightarrow 0$. To obtain uniform convergence with respect to $t_{k} \rightarrow 0$, we use a simple shift of the spectrum,

$$
e^{A_{k}}=e \cdot e^{B_{k}} \quad \text { for } B_{k}:=A_{k}-I,
$$

ensuring that $\lambda_{\min }\left(-B_{k}\right)=O(1)>0$. Now, we apply the quadrature (2.60) to the operator $B_{k}$, which leads to the uniform error estimate

$$
\left\|\exp \left(B_{k}\right)-\exp _{L}\left(B_{k}\right)\right\| \leq C e^{-s L / \log L} \quad \text { for all } k=-M, \ldots, N \text {, }
$$

where the constants $C, s>0$ do not depend on $L$ and $k$. With this procedure, we arrive at the following product quadrature.

Theorem 2.9. Under the conditions of Lemma 2.8, the expression

$$
\mathcal{Z}_{N, L}(\mathcal{L}):=2 h \sum_{k=-M}^{N} t_{k} \tanh \left(t_{k}\right) \mathcal{S}_{L, k}^{*} G \mathcal{S}_{L, k} \quad \text { with } \mathcal{S}_{L, k}:=\sum_{p=-L}^{L} c_{p} e^{1-z_{p}}\left(z_{p} I-B_{k}\right)^{-1}
$$

converges exponentially as $N, L \rightarrow \infty$,

$$
\left\|\mathcal{Z}(\mathcal{L})-\mathcal{Z}_{N, L}(\mathcal{L})\right\| \leq C\left[\left(1+\mu^{2}\right) e^{-\left(2 \pi \lambda_{0} d N\right)^{2 / 3}}+e^{-s L / \log L}\right] .
$$

Proof. The combination of the result of Lemma 2.8 with the modified quadrature (2.60) leads to the desired bound. 


\section{NeW QUADRATURE For the LyapunOV SOLUtion OPERATOR}

Quadrature Rule $I$ presented in the previous section contains a triple sum of elliptic resolvents (one sum from $\mathcal{Z}_{N, L}$ and two sums due to $\mathcal{S}_{L, k}$ and $\mathcal{S}_{L, k}^{*}$ ). In this section we propose a new scheme which contains only a double sum of resolvents.

3.1. Equations with elementary operators. Let $\mathcal{A}$ be a complex Banach algebra with identity $e$ and let $\mathcal{B}$ be a Banach algebra of operators on $\mathcal{A}$ considered as a Banach space. Given $\left\{U_{j}\right\},\left\{V_{j}\right\} \subset \mathcal{A}$, let $S \in \mathcal{B}$ be defined by

$$
S X:=\sum_{j=1}^{M} U_{j} X V_{j}
$$

where $\left\{U_{j}\right\}$ and $\left\{V_{j}\right\}$ are commutative subsets of $\mathcal{A}$ but $\left\{U_{j}\right\}$ need not commute with $\left\{V_{j}\right\}$. The operator $S$ (such operators are usually called elementary operators) was studied in [27] where it was shown that if $\Sigma(X, \mathcal{A})$ denotes the spectrum of $X \in \mathcal{A}$, then

$$
\Sigma(S, \mathcal{B}) \subset \sum_{j=1}^{M}\left\{\lambda \mu: \lambda \in \Sigma\left(U_{j}, \mathcal{A}\right), \mu \in \Sigma\left(V_{j}, \mathcal{A}\right)\right\}=: \Sigma_{U V} .
$$

Furthermore, if $f(\lambda)$ is holomorphic in a domain that contains $\Sigma_{U V}$, then there exist Cauchy domains $D_{i}^{V} \supset \Sigma\left(V_{i}, \mathcal{A}\right), D_{i}^{U} \supset \Sigma\left(U_{i}, \mathcal{A}\right), 1 \leq i \leq M$, such that for any $X \in \mathcal{A}$

$$
\begin{aligned}
f(S) X & =\frac{1}{(2 \pi i)^{M}} \int_{\partial D_{1}^{V}} \cdots \int_{\partial D_{M}^{V}} f\left(\sum_{j=1}^{M} \lambda_{j} U_{j}\right) X \prod_{j=1}^{M}\left(\lambda_{j} I-V_{j}\right)^{-1} d \lambda_{j} \\
& =\frac{(-1)^{M}}{(2 \pi)^{2 M}} \int_{\partial D_{1}^{V}} \cdots \int_{\partial D_{M}^{V}} \int_{\partial D_{1}^{U}} \cdots \int_{\partial D_{M}^{U}} f\left(\sum_{j=1}^{M} \lambda_{j} \mu_{j}\right) \\
& \times \prod_{j=1}^{M}\left(\mu_{j} I-U_{j}\right)^{-1} d \mu_{j} X \prod_{j=1}^{M}\left(\lambda_{j} I-V_{j}\right)^{-1} d \lambda_{j}
\end{aligned}
$$

where $\partial D_{i}^{V}, \partial D_{i}^{U}$ denote the boundaries of $D_{i}^{V}$ and $D_{i}^{U}$, respectively, and $I$ the unit operator. There are a number of papers dealing with invertibility and spectral properties of elementary operators (see, for example, [20, 21]).

Let us consider the operator equation

$$
S X=G
$$

and suppose that $S^{-1}$ exists. Then using formula (3.2) applied to the function

$$
f\left(\sum_{j=1}^{M} \lambda_{j} \mu_{j}\right) \equiv\left(\sum_{j=1}^{M} \lambda_{j} \mu_{j}\right)^{-1}
$$

we get

$$
X=\frac{(-1)^{M}}{(2 \pi)^{2 M}} \int_{\partial D_{1}^{V}} \ldots \int_{\partial D_{M}^{U}}\left(\sum_{j=1}^{M} \mu_{j} \lambda_{j}\right)^{-1} \prod_{j=1}^{M}\left(\mu_{j} I-U_{j}\right)^{-1} d \mu_{j} G \prod_{j=1}^{M}\left(\lambda_{j} I-V_{j}\right)^{-1} d \lambda_{j} .
$$


The following special case of equation (3.3) with bounded operators was considered in [2]:

$$
\sum_{j, k=0}^{n} c_{j k} A^{j} X B^{k}=Y,
$$

where $c_{j k} \in \mathbb{R}, \quad B \in \mathcal{L}\left(B_{1}\right), \quad A \in \mathcal{L}\left(B_{2}\right), \quad Y \in \mathcal{L}\left(B_{1}, B_{2}\right)$ are given bounded operators, $X \in \mathcal{L}\left(B_{1}, B_{2}\right)$ is the operator we are looking for, $B_{1}, B_{2}$ are Banach spaces, and $\mathcal{L}\left(B_{i}\right)$ and $\mathcal{L}\left(B_{1}, B_{2}\right)$ denote the sets of linear bounded operators acting in $B_{i}$ and from $B_{1}$ into $B_{2}$, respectively. It was shown that the unique solution is given by

$$
X=-\frac{1}{4 \pi^{2}} \oint_{\Gamma_{A}} \oint_{\Gamma_{B}} \frac{(A-\lambda I)^{-1} Y(B-\mu I)^{-1}}{P(\lambda, \mu)} d \lambda d \mu
$$

provided that $P(\lambda, \mu)=\sum_{j, k=0}^{n} c_{j k} \lambda^{j} \mu^{k} \neq 0$ for $(\lambda, \mu) \in \Sigma(A) \times \Sigma(B)$, where $\Sigma(A), \Sigma(B)$ are the spectral sets of $A$ and $B$, respectively, and $\Gamma_{A}$ and $\Gamma_{B}$ denote paths in the resolvent sets $\rho(A), \rho(B)$ of $A, B$ surrounding $\Sigma(A)$ and $\Sigma(B)$. The representations (3.5), (3.7) are useful in many applications (see, for example, [2, 18]) and can be justified also for unbounded operators provided the resolvents possess appropriate properties (see Lemma 3.1 below).

Particular cases of the equations (3.3), (3.6) are the Sylvester equation

$$
A X+X B=G
$$

and the Lyapunov equation

$$
A X+X A^{\top}=G .
$$

The Lyapunov (Sylvester) equation is involved, for example, while using Newton's method for solving Riccati matrix equations arising in optimal control problems 21, 25]. One has to solve the Riccati equation

$$
A X+X A^{\top}+X B B^{\top} X=-C^{\top} C
$$

for constructing a near-optimal reduced-order model for a dynamical system

$$
\begin{aligned}
& \dot{x}=A x+B u, \quad x(0)=x_{0}, \\
& y=C x
\end{aligned}
$$

with state $x \in \mathbb{R}^{n}$, input $u \in \mathbb{R}^{p}$ and output $y \in \mathbb{R}^{q}$ and with $A \in \mathbb{R}^{n \times n}, B \in \mathbb{R}^{n \times p}$, $C \in \mathbb{R}^{n \times q}$. Here the optimal control $u$ can be realised as $u(t)=-B^{\dagger} X x(t), t \in$ $(0, \infty)$.

Consider a control problem where the state is governed by partial differential equations. The spatial discretisation of the partial differential operator by a finiteelement or finite-difference method (yielding a system matrix $A$ ) leads to a system of ordinary differential equations of the type (3.10) with large and sparse matrices and finally to a Riccati equation which can be reduced to a sequence of Lyapunov equations. There are direct and iterative methods to solve the Lyapunov equation (cf. 22, 21, 22, 24, 26, 32]). The direct ones are preferred when solving equations with matrices of moderate size. Direct methods are often based on various decompositions of the matrix (e.g., the Schur decomposition) with complexity $\mathcal{O}\left(n^{3}\right)$, which restricts their use to problems with relatively small $n$. Iterative methods (SOR, ADI, multigrid and others) are applied to the Lyapunov equation when the 
matrix $A$ is large. In order to ensure computational stability and to decrease the number of iteration steps, various preconditioning techniques are used (cf. [23]).

The aim of this section is to find exponentially convergent approximations to the solutions of (3.3), 3.6). One can use these approximations to solve quadratic equations like the Riccati equation efficiently.

3.2. Exponentially convergent Quadrature Rule II. Below we consider a method for solving the Sylvester equation based on a Sinc quadrature.

We consider a strongly $P$-positive operators $C$ with spectral parabolae $P_{S, C}$ defined by

$$
\begin{aligned}
& \xi=\phi_{S, C}(s)=a_{p, C} s^{2}+b_{p, C}, \\
& \eta=\psi_{S, C}(s)=s,
\end{aligned}
$$

lying in the right half-plane of the complex plane and enveloped by the hyperbolic set $H_{I_{C}}$ defined by

$$
\begin{aligned}
z & =a_{I C} \cosh (\mu+i \nu)+i b_{I C} \sinh (\mu+i \nu) \\
& =a_{I C} \cosh \mu \cos \nu+i a_{e} \sinh \mu \sin \nu+i b_{I C}(\sinh \mu \cos \nu+i \cosh \mu \sin \nu) \\
& =\cosh \mu\left(a_{I C} \cos \nu-b_{I C} \sin \nu\right)+i \sinh \mu\left(a_{I C} \sin \nu+b_{I C} \cos \nu\right) \\
& =\sqrt{a_{I C}^{2}+b_{I C}^{2}}[\cos (\nu+\varphi) \cosh \mu+i \sin (\nu+\varphi) \sinh \mu] \\
& =\sqrt{a_{I C}^{2}+b_{I C}^{2}} \cosh [\mu+i(\nu+\varphi)],
\end{aligned}
$$

lying also in the right half-plane. We suppose that the set $H_{I_{C}}$ envelopes the spectral parabola $P_{S, C}$ for all $|\nu|<d_{C}$. The class of such operators $C$ will be called by $\mathbf{E}_{H_{C}}$.

Lemma 3.1. Let $A, B$ be operators of the classes $\mathbf{E}_{H_{A}}$ and $\mathbf{E}_{H_{B}}$. Choose the integration curves

$$
\Gamma_{I A}=\left\{\lambda=\xi+i \eta: \xi=a_{I A} \cosh s_{A}, \eta=b_{I A} \sinh s_{A}, s_{A} \in \mathbb{R}\right\}
$$

and

$$
\Gamma_{I B}=\left\{\mu=\xi+i \eta: \xi=a_{I B} \cosh s_{B}, \eta=b_{I B} \sinh s_{B}, s_{B} \in \mathbb{R}\right\}
$$

from the set (3.12) with $C=A$ or $C=B$ and $\nu=0$. Then the solution of the operator equation (3.8) can be represented by the Dunford-Cauchy integral

$$
X=-\frac{1}{4 \pi^{2}} \int_{\Gamma_{I A}} \int_{\Gamma_{I B}}(\lambda+\mu)^{-1}(\lambda I-A)^{-1} G(\mu I-B)^{-1} d \lambda d \mu
$$

or, after parametrisation, by

$$
X=-\frac{1}{4 \pi^{2}} \int_{-\infty}^{\infty} \int_{-\infty}^{\infty} F\left(s_{A}, s_{B}\right) d s_{A} d s_{B}
$$

where the integrand

$$
\begin{gathered}
F\left(s_{A}, s_{B}\right)=\frac{\lambda^{\prime}\left(s_{A}\right) \mu^{\prime}\left(s_{B}\right)}{\lambda\left(s_{A}\right)+\mu\left(s_{B}\right)}(\lambda I-A)^{-1} G(\mu I-B)^{-1}, \\
\lambda=\lambda\left(s_{A}\right)=a_{I A} \cosh s_{A}+i b_{I A} \sinh s_{A} \\
\mu=\mu\left(s_{B}\right)=a_{I B} \cosh s_{B}+i b_{I B} \sinh s_{B}
\end{gathered}
$$

can be estimated on the real axis by

$$
\|F(\eta, \zeta)\| \lesssim\|G\| e^{-\sqrt{a_{I A}}\left|s_{A}\right| / 2} e^{-\sqrt{a_{I B}}\left|s_{B}\right| / 2}
$$


Moreover, the integrand can be analytically extended into the strip $D_{d}$ of the width $d=\min \left\{d_{A}, d_{B}\right\}>0$ from (2.18) with $d_{A}, d_{B}$ defined analogously as in Lemma 2.3 and it belongs to the class $\mathbf{H}^{p}\left(D_{d}\right)$ for all $p \in[1, \infty]$ with respect to each variable.

Proof. Since the operators $A, B$ are of the class $\mathbf{E}_{H_{A}}$ and $\mathbf{E}_{H_{B}}$, due to (2.17) and due to

$$
\begin{aligned}
|\lambda+\mu| & =\sqrt{\left(a_{I A} \cosh s_{A}+a_{I B} \cosh s_{B}\right)^{2}+\left(b_{I A} \sinh s_{A}+b_{I B} \sinh s_{B}\right)^{2}} \\
& \geq a_{I A} \cosh s_{A}+a_{I B} \cosh s_{B} \\
& \geq \sqrt{a_{I A} a_{I B} \cosh s_{A} \cosh s_{B}}
\end{aligned}
$$

we get that the integrand $F\left(s_{A}, s_{B}\right)$ defined in 3.15) can be estimated on the real axis by

$$
\left\|F\left(s_{A}, s_{B}\right)\right\| \lesssim\|G\| e^{-\sqrt{a_{I A}}\left|s_{A}\right| / 2} e^{-\sqrt{a_{I B}}\left|s_{B}\right| / 2}
$$

The above estimate shows that both the integrals (3.13) and (3.14) converge.

The analyticity of the integrand can be violated only if one of the hyperbolic sets $H_{I_{A}}$ or $H_{I_{B}}$ intersects the corresponding spectral parabola. The latter is, with guarantee, not the case for $\nu<d=\min \left\{d_{A}, d_{B}\right\}$ (see also the proof of Lemma 2.3 and Figure 2). It follows from (3.16) that the integrand belongs to $\mathbf{H}^{p}\left(D_{d}\right)$ for all $p \in[0, \infty)$ with respect to each of the two variables. It is also easy to see that $\lambda+\mu \neq 0$ in both strips $D_{d_{A}}$ and $D_{d_{B}}$. The proof is complete.

3.3. Error analysis for Quadrature Rule II. In this section we use the notation from $\S 2.4 .1$. We will need the following lemma which can be proven similarly to Lemma 2.4 (see [7, 29]).

Lemma 3.2. For any operator-valued function $f \in \mathbf{H}^{1}\left(D_{d}\right)$, it holds that

$$
\eta(f, h)=\frac{i}{2} \int_{\mathbb{R}}\left\{\frac{f\left(\xi-i d^{-}\right) e^{-\pi(d+i \xi) / h}}{\sin [\pi(\xi-i d) / h]}-\frac{f\left(\xi+i d^{-}\right) e^{-\pi(d-i \xi) / h}}{\sin [\pi(\xi+d) / h]}\right\} d \xi
$$

providing the estimate

$$
\|\eta(f, h)\| \leq \frac{e^{-\pi d / h}}{2 \sinh (\pi d / h)}\|f\|_{\mathbf{H}^{1}\left(D_{d}\right)}
$$

If, in addition, $f$ satisfies on $\mathbb{R}$ the condition

$$
\|f(\xi)\| \leq c \exp (-\alpha|\xi|), \quad \alpha, c>0
$$

then taking $h=\sqrt{2 \pi d /(\alpha N)}$, we obtain

$$
\left\|\eta_{N}(f, h)\right\| \leq c_{1} e^{-\sqrt{2 \pi d \alpha N}}
$$

with a constant $c_{1}>0$ independent of $N$. 
Given integers $N_{A}, N_{B}$, we set

$$
\begin{aligned}
& h_{A}=2 \sqrt{\pi d_{A} /\left(\sqrt{a_{I A}} N_{A} / 2\right)} \asymp 1 / \sqrt{N_{A}}, \\
& h_{B}=2 \sqrt{\pi d_{B} /\left(\sqrt{a_{I B}} N_{B} / 2\right)} \asymp 1 / \sqrt{N_{B}}
\end{aligned}
$$

and approximate the integral (3.14) by the double quadrature sum

$$
X \approx X_{N_{A}, N_{B}}=h_{A} h_{B} \sum_{k=-N_{A}}^{N_{A}} \sum_{j=-N_{B}}^{N_{B}} F\left(k h_{A}, j h_{B}\right) .
$$

Due to Lemma 3.1 and Lemma 3.2 we have the following error estimate indicating exponential convergence of Quadrature Rule II.

Theorem 3.3. It holds that

$$
\left\|X-X_{N_{A}, N_{B}}\right\| \leq C e^{-\sqrt{\pi d_{A} \sqrt{a_{I A}} N_{A}}-\sqrt{\pi d_{B} \sqrt{a_{I B}} N_{B}}}
$$

with a constant $C>0$ independent of $N_{A}$ and $N_{B}$.

The exponential convergence of our quadrature rule allows us to introduce the following parallel algorithm to approximate the solution of the Sylvester (Lyapunov) equation with operators $A, B$ from the classes $\mathbf{E}_{H_{A}}$ and $\mathbf{E}_{H_{B}}$, respectively.

Algorithm 3.4. (a) Given $\varepsilon>0$, choose integers $N_{A}=O\left(\log ^{2} \varepsilon\right), N_{B}=O\left(\log ^{2} \varepsilon\right)$, set $h_{A}$ and $h_{B}$ as in (3.19) and determine

$$
\begin{array}{ll}
z_{A}\left(k h_{A}\right)=a_{I A} \cosh \left(k h_{A}\right)+i b_{I A} \sinh \left(k h_{A}\right) & \left(k=-N_{A}, \ldots, N_{A}\right), \\
z_{B}\left(k h_{B}\right)=a_{I B} \cosh \left(k h_{B}\right)+i b_{I B} \sinh \left(k h_{B}\right) & \left(j=-N_{B}, \ldots, N_{B}\right) .
\end{array}
$$

(b) Find the resolvents

$$
\begin{aligned}
& \left(z_{k} I-A\right)^{-1}, \quad k=-N_{A}, \ldots, N_{A}, \\
& \left(z_{j} I-B\right)^{-1}, \quad j=-N_{B}, \ldots, N_{B} .
\end{aligned}
$$

(c) Find the approximations $X_{N_{A}, N_{B}}$ for the solution $X$ of the Sylvester equation in the form (3.20.

Compared with Quadrature Rule I (see Theorem 2.9), the new Algorithm 3.4 includes only the double sum of the elliptic resolvents (it seems that it cannot be further improved).

To realise the constructive $\mathcal{H}$-matrix approximation, we build the $\mathcal{H}$-matrix representation for each individual elliptic resolvent (or its discrete version) involved into the representation (3.20). The latter sum contains $\left(2 N_{A}+1\right)\left(2 N_{B}+1\right)$ terms, where we set $N_{A}=O\left(\log ^{2} \varepsilon\right), N_{B}=O\left(\log ^{2} \varepsilon\right)$. The overall complexity of our Quadrature Rule II amounts to $O\left(n \log ^{q} n \log ^{4} \varepsilon\right)$, where $n$ is the problem size corresponding to the spacial discretisation to the elliptic operators $A$ and $B$. 


\section{Resolvent approximation to $\operatorname{sign}(H)$}

4.1. An exponential convergent quadrature rule. Let $t_{j}=j \pi / n, n=0, \ldots$, $2 n-1$, be an equidistant grid. Then for a given continuous function $f(x)$, the trigonometric polynomial

$$
u(t)=P_{n} f=\sum_{j=0}^{n} \alpha_{j} \cos (j t)+\sum_{j=1}^{n-1} \beta_{j} \sin (j t)
$$

with the coefficients

$$
\begin{aligned}
& \alpha_{0}=\frac{1}{2 n} \sum_{k=0}^{2 n-1} f_{k}, \quad f_{k}=f\left(t_{k}\right), \quad \alpha_{n}=\frac{1}{2 n} \sum_{k=0}^{2 n-1}(-1)^{k} f_{k}, \\
& \alpha_{j}=\frac{1}{n} \sum_{k=0}^{2 n-1} f_{k} \cos \left(j t_{k}\right), \quad \beta_{j}=\frac{1}{n} \sum_{k=0}^{2 n-1} f_{k} \sin \left(j t_{k}\right), \quad j=1, \ldots, n-1,
\end{aligned}
$$

is the trigonometric interpolant satisfying $u\left(t_{j}\right)=f_{j}, j=0, \ldots, 2 n-1$. Another representation of this polynomial is

$$
\left(P_{n} f\right)(t)=\sum_{k=0}^{2 n-1} f\left(t_{k}\right) L_{k}(t)=\frac{1}{2 n} \sin (n t) \sum_{k=0}^{2 n-1}(-1)^{k} f\left(t_{k}\right) \cot \frac{t-t_{k}}{2}
$$

with the Lagrange basis

$$
L_{j}(t)=\frac{1}{2 n} \sin n\left(t-t_{j}\right) \cot \frac{t-t_{j}}{2}, \quad t \neq t_{j}, \quad j=0, \ldots, 2 n-1 .
$$

If $f$ is analytic and $2 \pi$-periodic, then there exists a strip $D=\mathbb{R} \times(-s, s) \subset \mathbb{C}$ with $s>0$ such that $f$ can be extended to a holomorphic and $2 \pi$-periodic bounded function $f: D \rightarrow \mathbb{C}$ and the remainder in trigonometric interpolation can be estimated uniformly on $[0,2 \pi]$ by

$$
\left\|P_{n} f-f\right\|_{\infty} \leq M \frac{\operatorname{coth}(s / 2)}{\sinh (n s)},
$$

where $M$ denotes a bound for the holomorphic function $f$ on $D$. We can summarise this result by the estimate $\left\|P_{n} f-f\right\|_{\infty}=\mathcal{O}\left(e^{-n s}\right)$. Using the interpolant $P_{n} f$ instead of $f$, one gets the quadrature rule

$$
\int_{0}^{2 \pi} f(\tau) d \tau \approx \sum_{m=0}^{2 n-1} \gamma_{m} f_{m}
$$

with the quadrature coefficients

$$
\gamma_{m}=\int_{0}^{2 \pi} L_{m}(\tau) d \tau=\sum_{k=0}^{2 n-1}\left(\frac{\pi}{n} \delta_{m k}-(-1)^{k-m} \frac{\pi}{4 n^{2}}\right)=\pi / n
$$

Thus, in the case of analytic and $2 \pi$-periodic integrands, we arrive at a quadrature error $\mathcal{O}\left(e^{-n s}\right)$. 
4.2. Quadratures in the case of uniformly bounded operators. Let $H$ be a bounded operator, $\Gamma_{S}=\left\{z=z_{0}+r_{S} e^{i \phi} \in \mathbb{C}: \phi \in[0,2 \pi)\right\}, z_{0}=x_{0} \in \mathbb{R}_{+}, 0 \leq$ $r_{S}<x_{0}$, be the boundary of a disc $\Omega_{S}$ which contains the spectral set $\Sigma$ of $H$, and $\Gamma_{I}=\left\{z=z_{0}+r_{I} e^{i \phi} \in \mathbb{C}: \phi \in[0,2 \pi)\right\}, 0 \leq r_{I}<x_{0}$, be the integration path in (2.4). After parametrising the integral (2.4), we get

$$
\begin{aligned}
& \operatorname{sign}(H)=\frac{1}{\pi i} \int_{\Gamma_{I}}(z I-H)^{-1} d z-I=\frac{1}{\pi} \int_{0}^{2 \pi} F(\phi) d \phi-I, \\
& F(\phi)=r_{I} e^{i \phi}\left(\left(z_{0}+r_{I} e^{i \phi}\right) I-H\right)^{-1}, \quad \phi \in[0,2 \pi] .
\end{aligned}
$$

For a complex argument $\phi_{c}=\phi+i \psi$, the analyticity of the integrand

$$
F\left(\phi_{c}\right)=i r_{c} e^{i \phi}\left(\left(z_{0}+r_{R} e^{i \phi}\right) I-H\right)^{-1}, \quad r_{R}=r_{I} e^{-\psi},
$$

can be violated only if $\psi>0$, since in this case the resolvent circle $z=z_{0}+r_{R} e^{i \phi}$ lies inside of the spectral circle so that the resolvent can be unbounded. From the inequalities $r_{I}-\rho<r_{R}<x_{0}$ (this inequality guarantees that the resolvent circle lies outside the spectral one), where $\rho$ is the distance between the integration and the spectral circles, we get $-\ln \frac{x_{0}}{r_{I}}<\psi<\ln \frac{r_{I}}{r_{I}-\rho}$. Thus, the integrand $F(\phi)$ in (4.2) is a $2 \pi$-periodic function which can be holomorphically extended into the strip $S=\{w=\phi+i \psi: \phi \in(-\infty, \infty),|\psi|<s\}$, where $s=\min \left\{\ln \frac{x_{0}}{r_{I}}, \ln \frac{r_{I}}{r_{I}-\rho}\right\}$.

Now, applying the quadrature rule (4.1) to the integral (4.2), we get the following approximation of the operator sign function:

$$
\operatorname{sign}(H) \approx \operatorname{sign}_{N}(H):=\frac{1}{N} \sum_{k=0}^{2 N-1} F\left(\frac{k \pi}{N}\right)-I,
$$

where the quadrature error is $\mathcal{O}\left(e^{-N s}\right)$.

Remark 4.1. In applications, $A$ may be a finite-element or finite-difference approximation of a second order elliptic differential operator for which $r_{I}=\mathcal{O}\left(h^{-k}\right), k>0$, where $h$ is the spatial discretisation parameter. In this case we have $s=\ln \frac{r_{I}}{r_{I}-\rho}=$ $\ln \frac{1}{1-c h^{k}}=\mathcal{O}\left(h^{k}\right) \rightarrow 0$ as $h \rightarrow 0$ so that the error of the algorithm (4.3) is of the order $\mathcal{O}\left(e^{-c h^{k} N}\right)$ with some positive constant $c$. This leads to a polynomial complexity relying on the estimate $N=\mathcal{O}\left(h^{-k^{\prime}}\right)$ with $k^{\prime}>k$.

Due to Remark 4.1, for many applications we may need a special method that is robust with respect to $\operatorname{cond}(H)$.

4.3. Sinc-based approximation in the general case. Let $\Omega^{+}$be the set of eigenvalues of $H$ with positive real part and let $\Omega^{-}$be the corresponding set with negative real part. Consider the matrix-valued function $\mathcal{J}=\mathcal{J}(H)$ given by the integral representation

$$
\mathcal{J}=\frac{1}{\pi i} \int_{\Gamma}(\zeta I-H)^{-1} d \zeta=\operatorname{sign}(H)+I,
$$

where $\Gamma$ is the circle in the complex plane with the diameter $\left[x_{1}, x_{2}\right] \in \mathbb{R}, x_{1}, x_{2}>0$, which encloses $\Omega^{+}$. The parameter $\kappa:=x_{2} / x_{1}$ will be used in the following (it can be regarded as the condition number of $H$ ). We are interested in an accurate approximation of $\mathcal{J}(H)$ by the sum of a few resolvents $\left(z_{k} I-H\right)^{-1}$ with different parameters $z_{k}$. 
Under the substitution $\zeta \mapsto t:=c_{0}^{-1}\left(\zeta-\zeta_{0}\right)$ with $\zeta_{0}=\frac{x_{1}+x_{2}}{2}, c_{0}=\frac{x_{2}-x_{1}}{2}$, the integral $\mathcal{J}$ takes the form

$$
\mathcal{J}=\frac{1}{\pi i} \int_{\Gamma_{0}}(t I-B)^{-1} d t, \quad B=c_{0}\left(H-z_{0} I\right),
$$

where $\Gamma_{0}=\partial \mathcal{U}$ is the unit circle, while $\mathcal{U}$ is the unit disc centred at $t=0$. Next, we transform the integral over $\Gamma_{0}$ to an integral over the reference interval $K:=[-1,1]$. To that end we use the Zhukovski mapping

$$
z=\frac{1}{2}\left(t+\frac{1}{t}\right)
$$

which maps $z: \Gamma_{0} \rightarrow[-1,1]$. Denote by $\Omega_{z}^{+}, \Omega_{z}^{-} \in \mathbb{C}$ the respective images of $\Omega^{+}$ and $\Omega^{-}$under the mapping $t \mapsto z$. We also set $\Omega_{z}:=\Omega_{z}^{+} \cup \Omega_{z}^{-} \subset \mathbb{C}$.

It is worth noting that the transforms $t_{1}:=z+\sqrt{z^{2}-1}, t_{2}:=z-\sqrt{z^{2}-1}$ map $K$ onto the upper and lower halves of $\Gamma_{0}$, respectively. Therefore, since $d t=2 \frac{t^{2}}{t^{2}-1} d z$, the target integral over the two-sided slit curve $K$ can be written as

$$
\mathcal{J}(H)=\mathcal{J}_{1}(B)-\mathcal{J}_{2}(B)
$$

where

$$
J_{k}(B):=\frac{2}{\pi i} \int_{K}\left(t_{k}(z) I-B\right)^{-1} \frac{t_{k}^{2}(z)}{t_{k}^{2}(z)-1} d z, \quad k=1,2 .
$$

One can easily check that both functions $t_{k}(z), k=1,2$, have a regular behaviour as $z^{2} \rightarrow 1$ with $z \in K$, namely, $t_{1}(z) \rightarrow 1$ as $z \rightarrow 1$ and $t_{2}(z) \rightarrow-1$ as $z \rightarrow-1$. On the other hand, it can be shown by a simple calculation that

$$
\left|\frac{t_{1}^{2}(z)}{t_{1}^{2}(z)-1}-\frac{t_{2}^{2}(z)}{t_{2}^{2}(z)-1}\right| \leq \frac{c}{\sqrt{1-z^{2}}} \quad \text { as } \quad z^{2} \rightarrow 1, \Im m z=0 .
$$

Following 29, introduce the eye-shaped region (cf. Figure 4)

$$
\mathcal{D}:=\left\{z \in \mathbb{C}:\left|\arg \left(\frac{z+1}{z-1}\right)\right|<d\right\},
$$

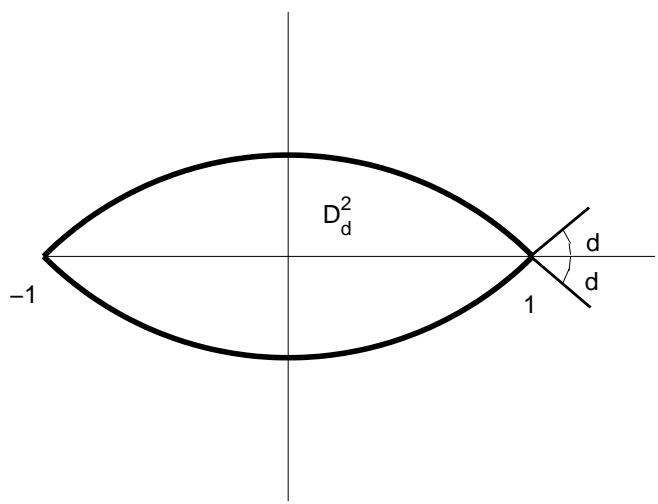

FIGURE 4. The eye-shaped region. 
where $d \in(0, \pi)$ is a given parameter. As in [29, Example 4.2.8], we define the functions

$$
\begin{gathered}
w=\Phi(z):=\log \frac{z-a}{b-z}, \\
\Phi^{\prime}(z)=\frac{b-a}{(z-a)(b-z)},
\end{gathered}
$$

and the corresponding sequence of collocation points

$$
z_{k}=\frac{a+b e^{k \delta}}{1+e^{k \delta}}
$$

where $\delta>0$ is the grid parameter. In our particular example, we set $a=-1$, $b=1$. As a matter of fact, $w$ is a conformal map of $\mathcal{D}$ onto the strip $D_{d}$ defined by (2.18). If $d \in(0, \pi)$ the class $\mathbf{L}_{\alpha, \beta}(\mathcal{D})$ is defined as the family of all matrix-valued functions $F$ which are holomorphic in $\mathcal{D}$ and which for some constant $C>0$ satisfy the inequality

$$
\|F(z)\| \leq C|z-a|^{\alpha}|b-z|^{\beta} \quad \text { in } \mathcal{D} .
$$

We rewrite our integral in the form

$$
\mathcal{J}(B)=\int_{K} F(z) d z
$$

with

$$
F(z)=\frac{2}{\pi i}\left[\left(t_{1}(z) I-B\right)^{-1} \frac{t_{1}^{2}(z)}{t_{1}^{2}(z)-1}-\left(t_{2}(z) I-B\right)^{-1} \frac{t_{2}^{2}(z)}{t_{2}^{2}(z)-1}\right] .
$$

Theorem 4.2. Assume that $\mathcal{D} \cap \Omega_{z}=\emptyset$ and let $N \in \mathbb{N}$ be given. Choose the parameter $\delta=\left(\frac{4 \pi d}{N}\right)^{1 / 2}$ in (4.7). Then there exists a constant $C_{0}$, independent of $N$, such that

$$
\left\|\int_{K} F(z) d z-\delta \sum_{k=-N}^{N} \frac{F\left(z_{k}\right)}{\Phi^{\prime}\left(z_{k}\right)}\right\| \leq C_{0} e^{-(\pi d N)^{1 / 2}},
$$

where $\Phi^{\prime}$ and $z_{k}$ are given by (4.6), (4.7) with $a=-1, b=1$.

Proof. The assumption that $\mathcal{D} \cap \Omega_{z}=\emptyset$ combined with (4.5) implies that $F \in$ $\mathbf{L}_{1 / 2,1 / 2}(\mathcal{D})$. Now the results of [29, Example 4.2.8] (see also Theorem 4.2.6 therein) modified by the usual arguments (see 6] and 7] for more details) to the case of matrix-valued functions lead to the desired exponential convergence.

It can be shown that the constant $C_{0}$ in (4.8) can be estimated by

$$
C_{0} \leq C \int_{\partial \mathcal{D}}\|F(z)\||d z|,
$$

where $C$ does not depend on $F$. If $H$ represents the finite-element stiffness matrix for the second order elliptic operator, then we can derive $C_{0}=\mathcal{O}(\kappa)=\mathcal{O}\left(h^{-2}\right)$, where $h>0$ is the corresponding mesh size. Therefore, a quadrature error of order $\varepsilon>0$ can be achieved with $N=\mathcal{O}\left(|\log h|^{2}+|\log \varepsilon|^{2}\right.$ ) (compare with the number of Newton's iteration in [11] to compute $\operatorname{sign}(H)$ alternatively). 
We conclude the paper with the following remark that describes the uniform convergence of the quadrature rule for the matrix exponential $e^{-t \mathcal{L}}$ with respect to $t \geq 0$.

Remark 4.3. Our analysis of the quadratures in Theorem 2.5 indicates that the approximation to the integral (2.20) is no longer uniform in $t \in[a, \infty)$ if $a=0$. It is remarkable that in the limit case $t=0$ the target integral is nothing but $1 / 2 \mathcal{J}(\mathcal{L})$ with $\mathcal{J}$ given by (4.4). Therefore, assuming that $\mathcal{L} \in \mathbb{R}^{n \times n}$, we can apply a similar quadrature to the integral (2.20) as in (4.8) just by substituting $(\zeta I-H)^{-1}$ by the corresponding ansatz $e^{-\zeta t}(\zeta I-H)^{-1}$. Clearly, all assumptions of Theorem 4.2 are valid for the new integrand and we arrive at the same approximation result as in (4.8), now uniformly in $t \geq 0$. Again the number of terms can be estimated by $N=\mathcal{O}\left(|\log (\operatorname{cond}(\mathcal{L}))|^{2}+|\log \varepsilon|^{2}\right)$, where one can expect $\operatorname{cond}(\mathcal{L})=\mathcal{O}\left(h^{-2}\right)$ for quasi-uniform meshes.

\section{REFERENCES}

1. K.I. Babenko: Foundations of the Numerical Analysis. Nauka, Moscow, 1986 (in Russian). MR 88g:65001

2. R. Bartels and G.W. Stewart: Algorithm 432: Solution of the matrix equation $A X+X B=C$. Comm. ACM 15 (1972), 820-826.

3. R. Bellman: Introduction to Matrix Analysis. McGraw-Hill Book Company Inc., New York, 1960. MR 23:A153

4. I.P. Gavrilyuk: Strongly P-positive operators and explicit representation of the solutions of initial value problems for second order differential equations in Banach space. J. Math. Anal. Appl. 236 (1999), 327-349. MR 2001j:34072

5. I.P. Gavrilyuk, W. Hackbusch and B.N. Khoromskij: $\mathcal{H}$-Matrix approximation for the operator exponential with applications. Numer. Math. 92 (2002), 83-111. MR 2003g:65061

6. I.P. Gavrilyuk, W. Hackbusch and B.N. Khoromskij: $\mathcal{H}$-Matrix approximation for elliptic solution operators in cylinder domains. East-West J. of Numer. Math. 9 (2001) 25-59. MR 2002e:65064

7. I.P. Gavrilyuk, W. Hackbusch and B.N. Khoromskij: Data-sparse approximation to operatorvalued functions of elliptic operators. Math. Comp., 73 (2004) 1297-1324.

8. I.P. Gavrilyuk, W. Hackbusch and B.N. Khoromskij: Data-sparse approximation to a hierarchy of operator-valued functions. In: Proceedings of the 18th GAMM-Seminar Leipzig 2002, 31-52 (ISBN 3-00-009258-7, http://www.mis.mpg.de/conferences/gamm/2002).

9. I.P. Gavrilyuk and V.L. Makarov: Exponentially convergent parallel discretization methods for the first order evolution equations, Appl. Math. Inform. 5 (2000), 47-69, 79 (2001).

10. L. Grasedyck and W. Hackbusch: Construction and arithmetics of $\mathcal{H}$-matrices. Computing 70 (2003), 295-334.

11. L. Grasedyck, W. Hackbusch and B.N. Khoromskij: Solution of large scale algebraic matrix Riccati equations by use of hierarchical matrices. Computing 70 (2003), 121-165.

12. W. Hackbusch: A sparse matrix arithmetic based on $\mathcal{H}$-matrices. Part I: Introduction to $\mathcal{H}$-matrices. Computing 62 (1999), 89-108. MR 2000c:65039

13. W. Hackbusch and B. N. Khoromskij: A sparse $\mathcal{H}$-matrix arithmetic. Part II: Application to multi-dimensional problems. Computing 64 (2000), 21-47. MR 2001i:65053

14. W. Hackbusch and B. N. Khoromskij: A sparse $\mathcal{H}$-matrix arithmetic: General complexity estimates. J. Comp. Appl. Math. 125 (2000), 479-501. MR 2002f:65151

15. W. Hackbusch and B.N. Khoromskij: Towards $\mathcal{H}$-matrix approximation of the linear complexity. Operator Theory: Advances and Applications, Vol. 121, Birkhäuser Verlag, Basel, 2001, 194-220. MR 2002f:65203

16. W. Hackbusch and B.N. Khoromskij: Hierarchical Kronecker tensor-product approximation to a class of nonlocal operators in high dimensions. Preprint MPI MIS, No. 16, Leipzig 2004; Computing (to appear).

17. M. Hochbruck and C. Lubich: On Krylov subspace approximations to the matrix exponential operator. SIAM J. Numer. Anal. 34 (1997) 1911-1925. MR 98h:65018 
18. Ju. L. Dalezkij and M.G. Krein: Stability of solutions of differential equations in Banach space. Nauka, Moscow, 1970 (in Russian) MR 50:5125

19. L.A. Fialkow: Spectral properties of elementary operators, Part I. Acta Sci. Math. 46 (1983), 269-282. MR 85h:47003

20. L.A. Fialkow: Spectral properties of elementary operators, Part II. Trans. Amer. Math. Soc. 290 (1985), 415-429. MR 86j:47005

21. Z. Gajić and M.T.J. Qureshi: Lyapunov Matrix Equation in System Stability and Control. Academic Press, San Diego, 1995. MR 96g:93001

22. G.H. Golub, S. Nash. and C. Van Loan: A Hessenberg-Schur method for the problem AX + $X B=C$. IEEE Trans. Automat. Cont. 24 (1979), 909-913. MR 81a:65046

23. M. Hochbruck and G. Starke: Preconditioned Krylov subspace methods for Lyapunov matrix equations. SIAM J. Matrix Anal. Appl. 16 (1995), 156-171. MR 95k:65045

24. P. Lancaster: Explicit solutions of linear matrix equations. SIAM Review 12 (1970), 544-566. MR 43:4841

25. I. Lasiecka and R. Triggiani: Algebraic Riccati equations arising in boundary/point control: A review of theoretical and numerical results, I: Continuous case. In: Perspectives in Control Theory, Birkhäuser, Basel, 1990, 175-210. MR 91e:93019

26. A. Lu and E.L. Wachspress: Solution of Lyapunov equations by ADI iteration. Comp. Math. Appl. 21 (1991), 43-58.

27. G. Lumer and M. Rosenblum: Linear operator equations. Proc. Amer. Math. Soc. 10 (1959), 32-41. MR 21:2927

28. D. Sheen, I. H. Sloan and V. Thomée: A parallel method for time-discretization of parabolic problems based on contour integral representation and quadrature, Math. Comp. 69 (2000), 177-195. MR 2000i:65161

29. F. Stenger: Numerical methods based on Sinc and analytic functions. Springer Verlag, 1993. MR 94k:65003

30. F. Stenger: Collocating convolutions. Math. Comp., 64 (1995) 211-235. MR 95c:65038

31. F. Stenger, B. Barkey and R. Vakili: Sinc convolution method of solution of Burgers' equation. In "Computation and Control III" (K. Bowers and J. Lund, eds.), Birkhäuser, Basel, 1993, 341-354. MR 94e:93007

32. E.L. Wachspress: Iterative solution of the Lyapunov matrix equation. Appl. Math. Lett. 1 (1988) 87-90.

Berufsakademie Thüringen, Am Wartenberg 2, D-99817 Eisenach, Germany

E-mail address: ipg@ba-eisenach.de

Max-Planck-Institute for Mathematics in the Sciences, Inselstr. 22-26, D-04103 LeipZig, Germany

E-mail address: wh@mis.mpg.de

Max-Planck-Institute for Mathematics in the Sciences, Inselstr. 22-26, D-04103 LEIPZIG, GERMANY

E-mail address: bokh@mis.mpg.de 